\title{
High Rates of Return to Sports Activities and Work After Osteotomies Around the Knee: A Systematic Review and Meta-Analysis
}

\author{
Alexander Hoorntje ${ }^{1,2} \cdot$ Suzanne Witjes $^{1,2} \cdot$ P. Paul F. M. Kuijer ${ }^{3} \cdot$ Koen L. M. Koenraadt $^{1}$. \\ Rutger C. I. van Geenen ${ }^{1} \cdot$ Joost G. Daams $^{4} \cdot$ Alan Getgood $^{5} \cdot$ Gino M. M. J. Kerkhoffs $^{2}$
}

Published online: 11 April 2017

(C) The Author(s) 2017. This article is an open access publication

\begin{abstract}
Background Knee osteotomies are proven treatment options, especially in younger patients with unicompartmental knee osteoarthritis, for certain cases of chronic knee instability, or as concomitant treatment for meniscal repair or transplantation surgery. Presumably, these patients wish to stay active. Data on whether these patients return to sport (RTS) activities and return to work (RTW) are scarce. Objectives Our aim was to systematically review (1) the extent to which patients can RTS and RTW after knee osteotomy and (2) the time to RTS and RTW.

Methods We systematically searched the MEDLINE and Embase databases. Two authors screened and extracted data, including patient demographics, surgical technique, pre- and postoperative sports and work activities, and confounding factors. Two authors assessed methodological quality. Data on pre- and postoperative participation in sports and work were pooled.
\end{abstract}

Electronic supplementary material The online version of this article (doi:10.1007/s40279-017-0726-y) contains supplementary material, which is available to authorized users.

Gino M. M. J. Kerkhoffs

g.m.kerkhoffs@amc.uva.nl

1 Department of Orthopaedic Surgery, Amphia Hospital, Foundation FORCE (Foundation for Orthopedic Research Care and Education), Molengracht 21, 4818 CK Breda, The Netherlands

2 Department of Orthopaedic Surgery, Academic Medical Center, ACES (Academic Centre for Evidence-based Sports medicine), ACHSS (Amsterdam Collaboration for Health and Safety in Sports), Meibergdreef 9, 1105 AZ Amsterdam, The Netherlands
Results We included 26 studies, involving 1321 patients (69\% male). Mean age varied between 27 and 62 years, and mean follow-up was 4.8 years. The overall risk of bias was low in seven studies, moderate in ten studies, and high in nine studies. RTS was reported in 18 studies and mean RTS was $85 \%$. Reported RTS in studies with a low risk of bias was $82 \%$. No studies reported time to RTS. RTW was reported in 14 studies; mean RTW was $85 \%$. Reported RTW in studies with a low risk of bias was $80 \%$. Time to RTW varied from 10 to 22 weeks. Lastly, only 15 studies adjusted for confounders.

Conclusion Eight out of ten patients returned to sport and work after knee osteotomy. No data were available on time to RTS. A trend toward performing lower-impact sports was observed. Time to RTW varied from 10 to 22 weeks, and almost all patients returned to the same or a higher workload.
3 Coronel Institute of Occupational Health, Academic Medical Center, University of Amsterdam, Meibergdreef 9, 1105 AZ Amsterdam, The Netherlands

4 Medical Library, Academic Medical Center, University of Amsterdam, Meibergdreef 9, 1105 AZ Amsterdam, The Netherlands

5 Department of Orthopaedic Surgery, Fowler Kennedy Sport Medicine Clinic, Western University, 3M Centre, 1151 Richmond Street, London, ON N6A 3K7, Canada 


\section{Key Points}

Most patients return to sports activities after knee osteotomy, with a tendency to lower-impact sports, and most patients return to work at the same or an even higher workload.

Systematic comparison of current literature is hampered by heterogeneity in patient populations, operative techniques, and the overall lack of accounting for possible confounding factors such as physical and mental comorbidities, preoperative sports level and work status, patient motivation, and surgeon's advice.

Future prospective studies are needed to gain better insight into the reasons patients do not return to sport or work. These studies should correct for confounders and use the pre-symptomatic phase as a reference point when assessing return to sport and work.

\section{Introduction}

Osteotomies around the knee, such as high tibial osteotomy (HTO) and distal femoral osteotomy (DFO), are well-accepted procedures for the treatment of early-stage unicompartmental knee osteoarthritis (OA) due to varus- or valgus malalignment [1-3]. With the rise of knee arthroplasty (KA) surgery in the 1970s, use of these procedures declined rapidly [4,5] as osteotomies were considered more demanding than KA and the outcomes and complications less predictable [4]. However, KAs clearly also have their limitations, especially for younger patients in terms of the low percentage of patients returning to highimpact activities, and the possible higher risk of polyethylene wear if they do $[6,7]$. Thus, since patients with knee $\mathrm{OA}$ are becoming younger and wish to perform more demanding high activities [8, 9], osteotomies around the knee have gained renewed attention. The current thought is that a knee osteotomy may postpone or even avoid KA and presumably allow patients to return to more demanding activities, since native joint structures are preserved.

In addition to the high demands of present-day patients, several other reasons exist for the renewed attention on and increased use of osteotomies around the knee. Outcomes from HTO and DFO have significantly improved with new operative techniques, improved fixation devices, updated evidence-based guidelines, and careful patient selection $[4,10,11]$. As a result, several studies have demonstrated distinct relief of pain and significant functional improvements after HTO and DFO [2, 4, 12]. Survival rates of $87-99 \%$ at 5 years and $66-84 \%$ at 10 years have been reported for HTO [13-15] and of 74-90\% at 5 years $[16,17]$ and $64-82 \%$ at 10 years [18-20] for DFO. Given these good results, it is reasonable to first consider a knee osteotomy when indication criteria are suitable [4, 21].

Indications for osteotomies have also been extended. In addition to the treatment of unicompartmental OA, osteotomies around the knee are increasingly performed as a concomitant treatment to correct alignment in ligament reconstruction, articular cartilage restoration procedures, and meniscal repair or transplantation surgery [22-26]. In these patients, who are mostly younger and more active, the function of the osteotomy is to (1) reduce strain on the reconstructed ligament graft or the posterolateral corner in cases of varus alignment or (2) unload the involved compartments and thereby reduce stress to the biological repair tissue and potentially prevent or postpone progression of early knee OA. Good results for these combined procedures in terms of functional outcome and survival have also been reported [23, 26].

Thus, osteotomies around the knee are increasingly performed in younger patients and show good results in unicompartmental $\mathrm{OA}$ and in reconstructive knee surgery. Johnstone et al. [27] suggested that, if osteotomies are being promoted for younger patients, it is important that they perform well in terms of return to sport (RTS) and return to work (RTW). However, studies that report on RTS and RTW after osteotomies around the knee are sparse, and a clear message is lacking in the literature. Consequently, the actual extent to which patients RTS and RTW is still largely unknown. Therefore, the purpose of the present study was to systematically summarize the available evidence on the extent to which patients RTS and RTW after osteotomies around the knee as well as timing of the return.

\section{Methods}

\subsection{Search Strategy}

We used the PRISMA (Preferred Reporting Items for Systematic reviews and Meta-Analyses) guidelines for this systematic review [28]. Before commencing the literature search, a research protocol was developed and agreed upon by all authors. This protocol was published online at the PROSPERO International prospective register of systematic reviews (http://www.crd.york.ac.uk/PROSPERO/; registration number CRD42016029929). The clinical librarian (JD) developed the search strategy in close cooperation with the first author $(\mathrm{AH})$. We used the World Health Organization International Clinical Trials Registry 
Platform (WHO-ICTRP) database to identify relevant search terms and to search for ongoing clinical trials on our subject. We searched the electronic databases MEDLINE via PubMed and Embase via OvidSP for relevant literature and the Cochrane database for systematic reviews. Searches were performed up until 21 September 2016. In all databases, the following four categories of keywords and related synonyms were used to build a sensitive search strategy and to provide a systematic search: osteotomy, sport, work, and recovery of function. Search terms were truncated using an asterisk (*) to find all terms beginning with a specific word. Within each keyword category, the different synonyms were combined using the Boolean command "OR" and categories were linked with the Boolean command "AND". The exact details of the search strategy can be found in the Electronic Supplementary Material (ESM) Appendix S1. The reference lists of selected studies were screened to identify additional studies for inclusion. We also performed a forward search using Web of Science to see which of these studies had been referred to by other authors after publication.

\subsection{Eligibility Criteria and Study Selection}

We used the Rayyan screening tool for systematic reviews to screen titles and abstracts [29]; all abstracts were screened by two independent reviewers (AH, PK). Discrepancies were resolved by discussion; where there was doubt, the article was included in the full-text screening process. One author (AH) then selected suitable studies based on the eligibility criteria established in the research protocol. This selection was then reviewed by a second author (SW), and discrepancies were resolved by discussion. Inclusion criteria were as follows: observational or intervention studies describing patients with malalignment who underwent any type of corrective knee osteotomy for any indication and who were participating in sport activities and/or working before the surgery and intended to RTS and/or RTW after surgery. No language restrictions were used. The primary outcomes were the percentage and number of patients to RTS and RTW, preferably described in terms of level, duration, and frequency. Secondary outcomes included activity-specific outcome measures, namely the Tegner activity score (0-10; higher is better), the Lysholm score (0-100; higher is better), the International Knee Documentation Committee (IKDC) objective score (0-100; higher is better), the University of California, Los Angeles (UCLA) activity score (0-10; higher is better), and the Naal activity score, which investigates preand postoperative engagement in 20 different sports activities. The Reichsausschuss für Arbeitszeitermittlung (REFA; German workload classification) Association classification system (from " $0=$ work with no physical strain" to " $4=$ work with most heavy physical strain") was also collected as a work-related outcome measure.

\subsection{Methodological Quality}

We assessed the risk of bias of the included studies using the Quality in Prognosis Studies (QUIPS) tool [30]. This qualityassessment tool includes six domains of potential bias: (1) study participation, (2) study attrition, (3) prognostic factor measurement, (4) outcome measurement, (5) study confounding, and (6) statistical analysis and reporting. Each domain contains two or more sub-domains that should be rated as "yes," "partial," "no," or "unsure." The answers to each sub-domain are then combined, leading to a "low," "moderate," or "high" risk of bias. The first author $(\mathrm{AH})$ assessed the quality of all included studies; this was then repeated independently by two other authors (PK, KK), who each assessed the risk of bias for half of the included studies. Disagreements were resolved by discussion and, if necessary, involving a third reviewer. The details of the quality assessment can be found in the ESM Appendix S2. We considered a study to have an overall low risk of bias when the methodological risk of bias was rated as low or moderate in all six domains, with at least four domains rated as low. A study was rated as having an overall high risk of bias if two or more of the domains were scored as high. In-between quality was scored as moderate. Results of the studies with a low risk of bias are discussed in the text and those of the studies with a moderate or high risk of bias are presented in the data extraction table (Table 1).

\subsection{Data Extraction}

One author $(\mathrm{AH})$ extracted data from all selected original studies, and this was independently repeated by one other author (SW). Disagreements were resolved by discussion. The authors used a standardized data extraction form that included the following: (1) study information: author, year, country, and reference number; (2) study design and follow-up; (3) information about study population: cohort, population size, sex, age, body mass index (BMI), comorbidities; (4) description of rehabilitation protocols used; (5) definition of outcome measures; (6) preoperative activity and definition (e.g., pre-symptomatic or at time of surgery); (7) postoperative activity; (8) RTS and RTW percentages and time to RTS and RTW; (9) confounding factors taken into account for RTS and RTW, such as age, sex, BMI, restricting comorbidities, complications, preoperative sports or work level, surgeon advice, or psychosocial factors. Authors were contacted if data were missing or only available in graphs. If this information was not provided, available data were read off the graphs. 


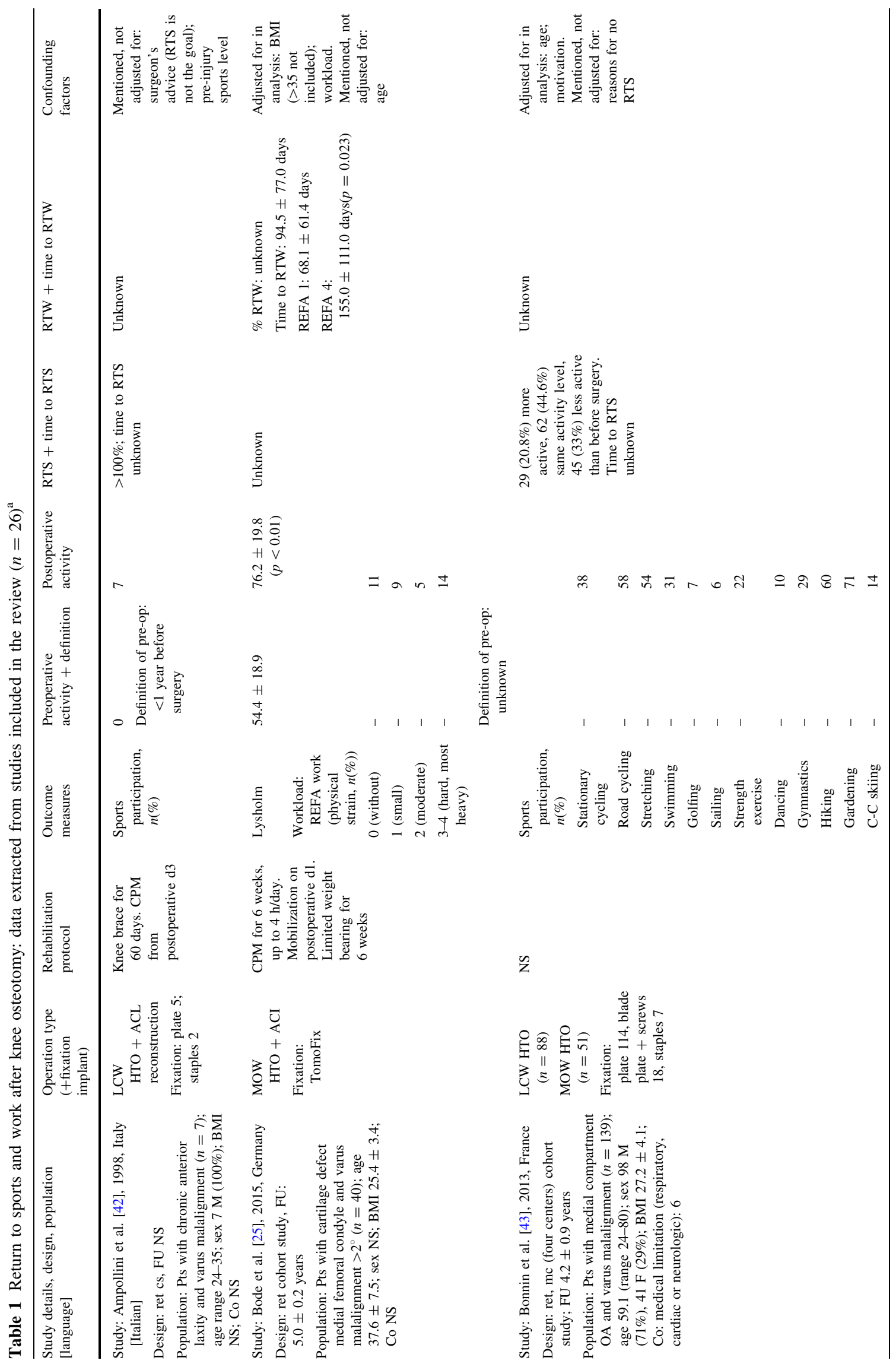




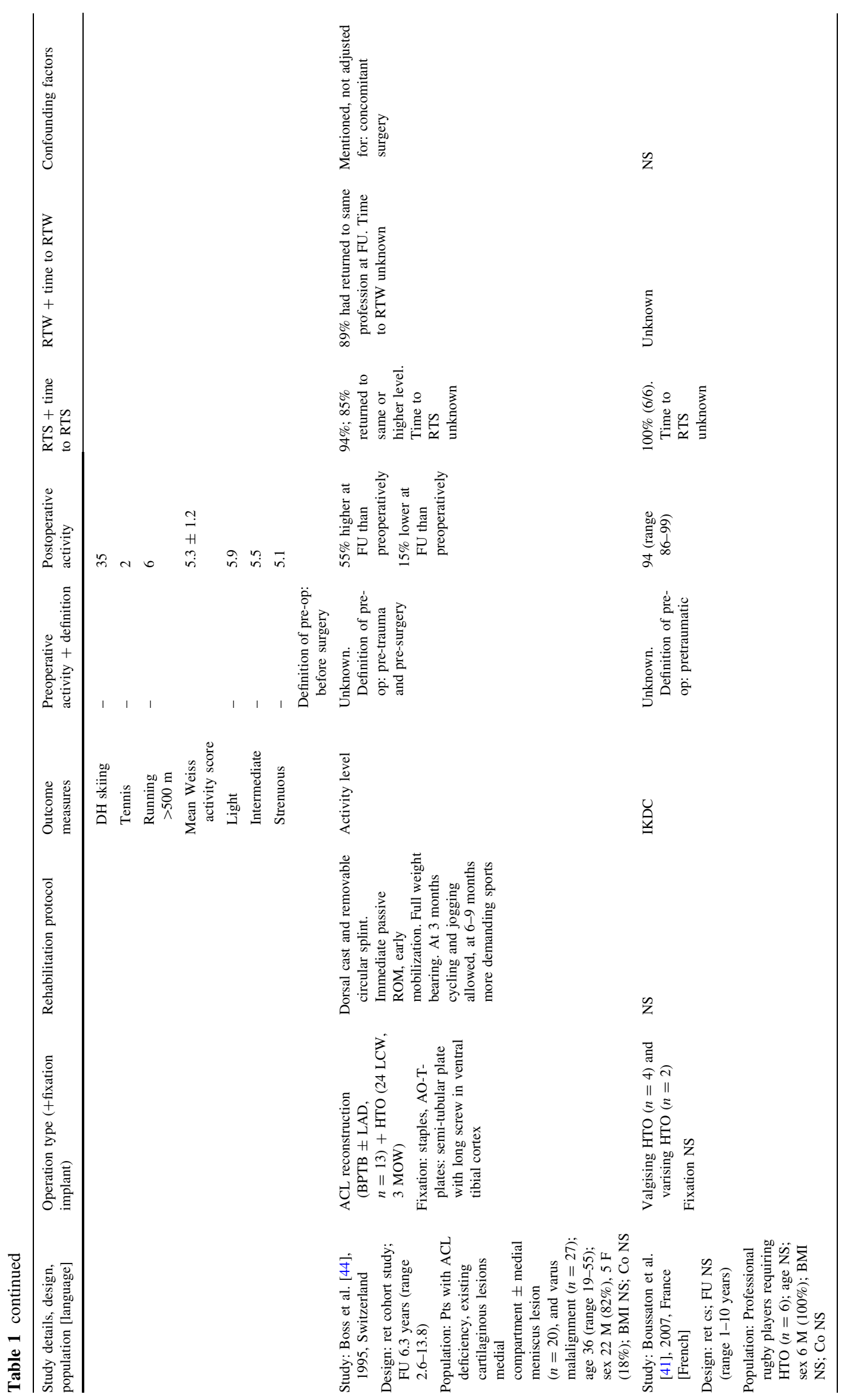




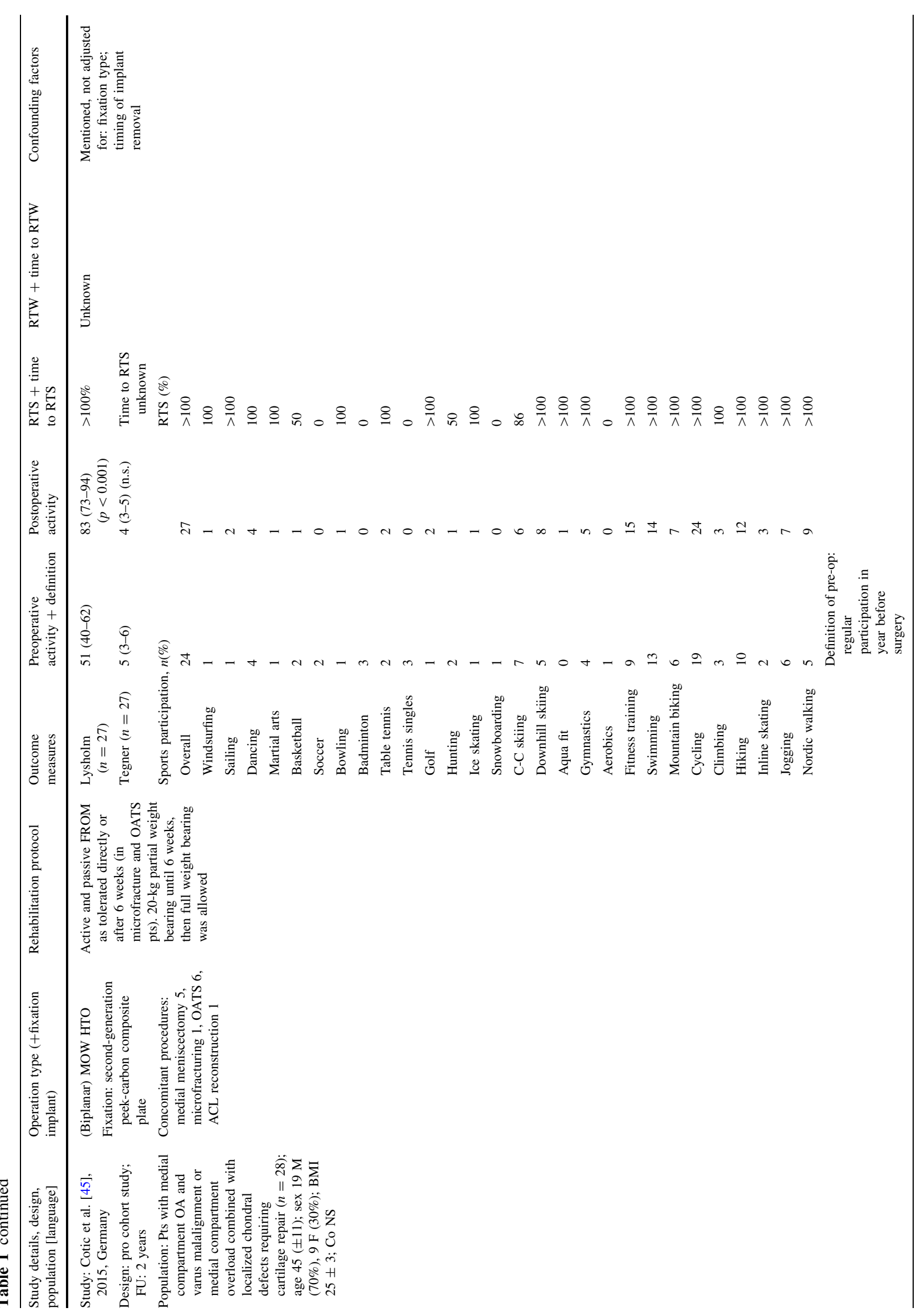




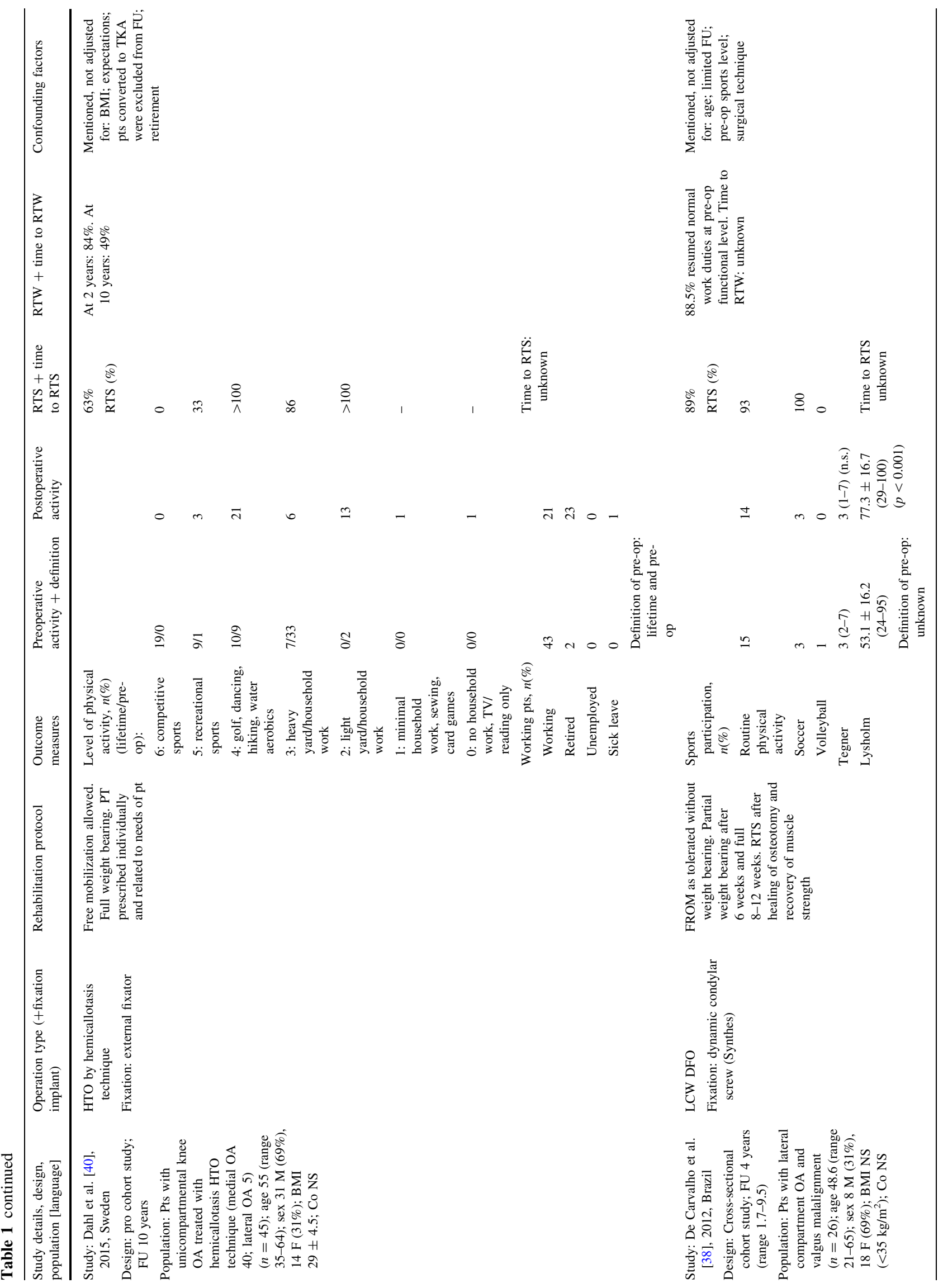




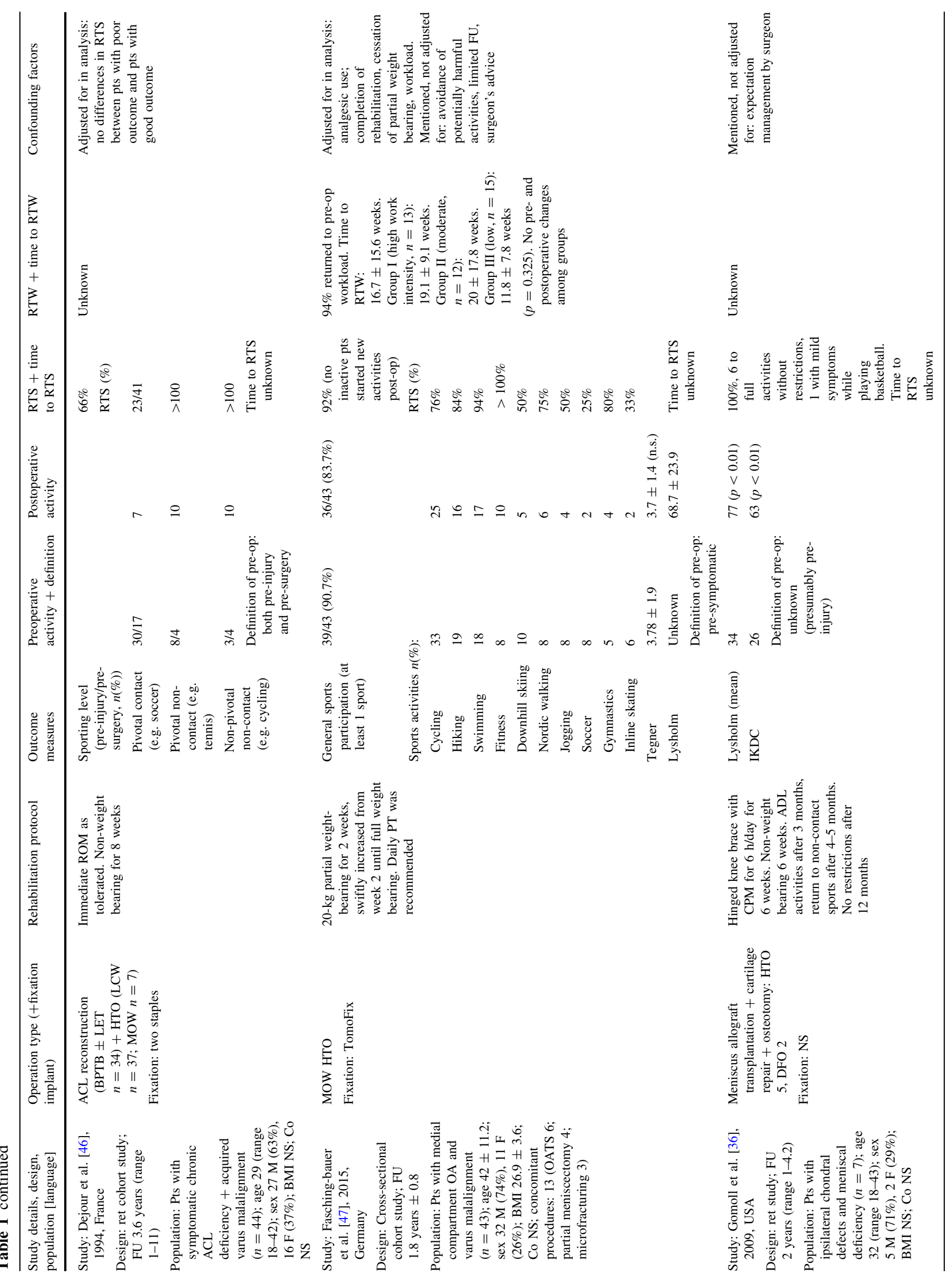




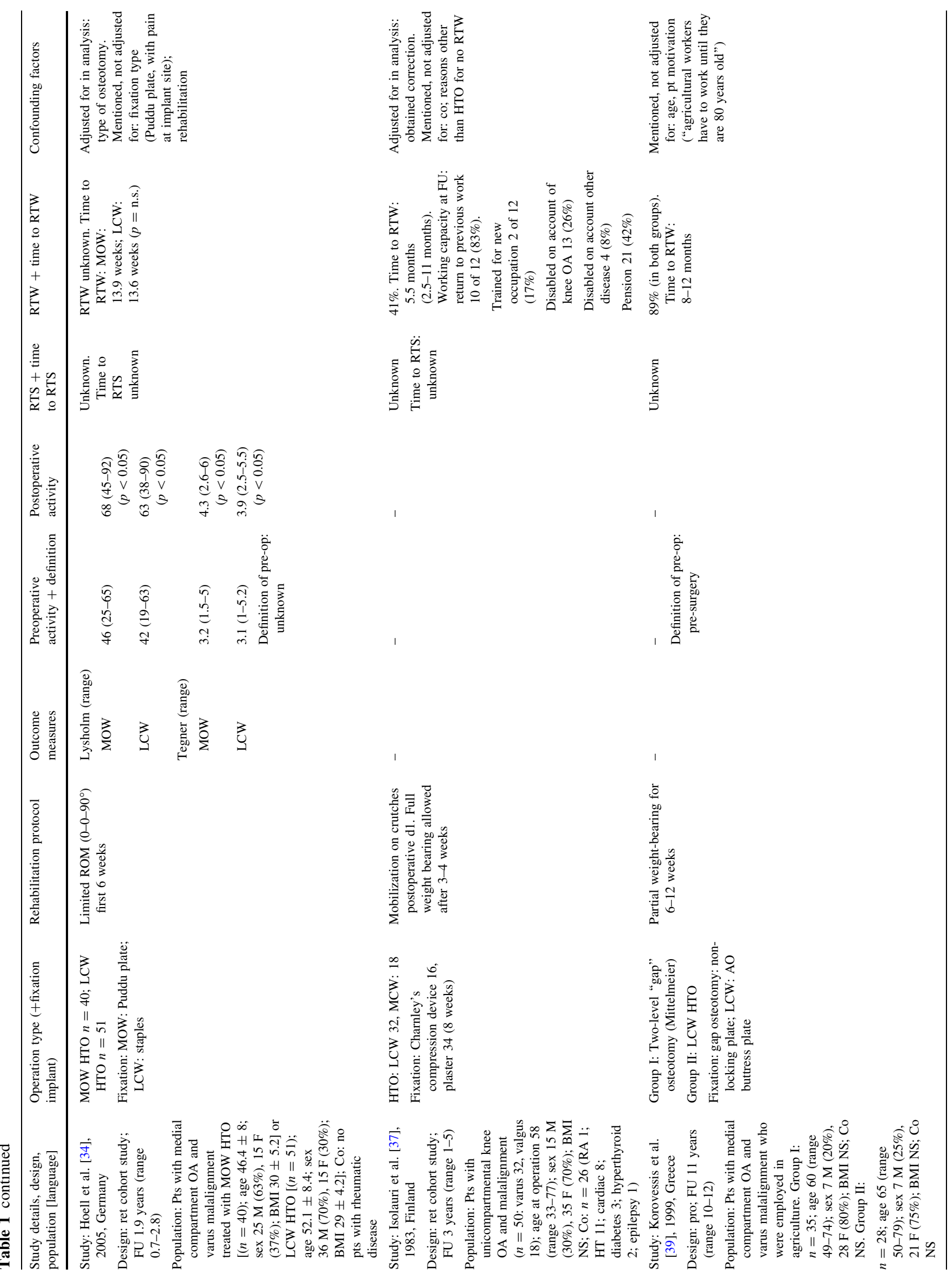




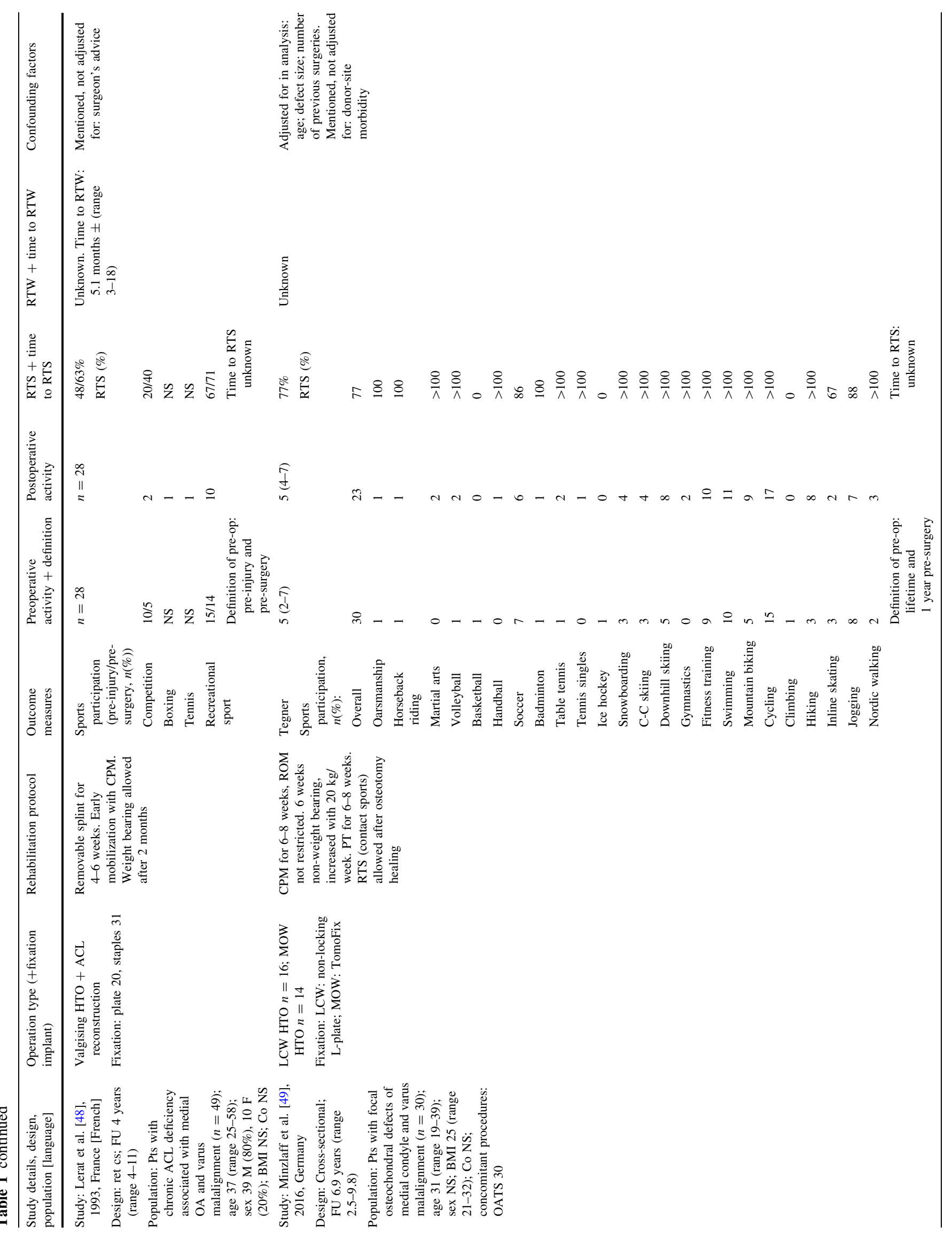




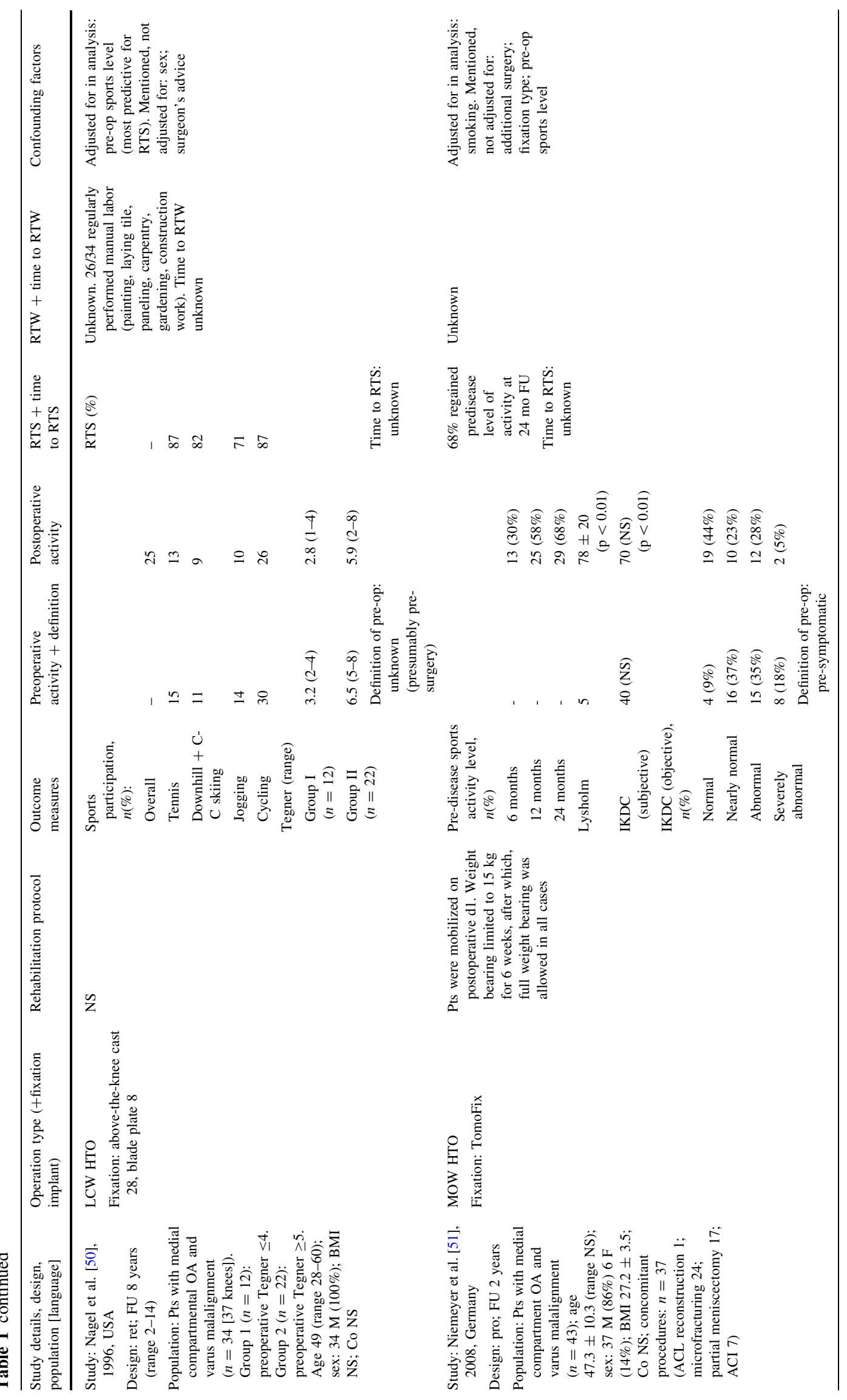




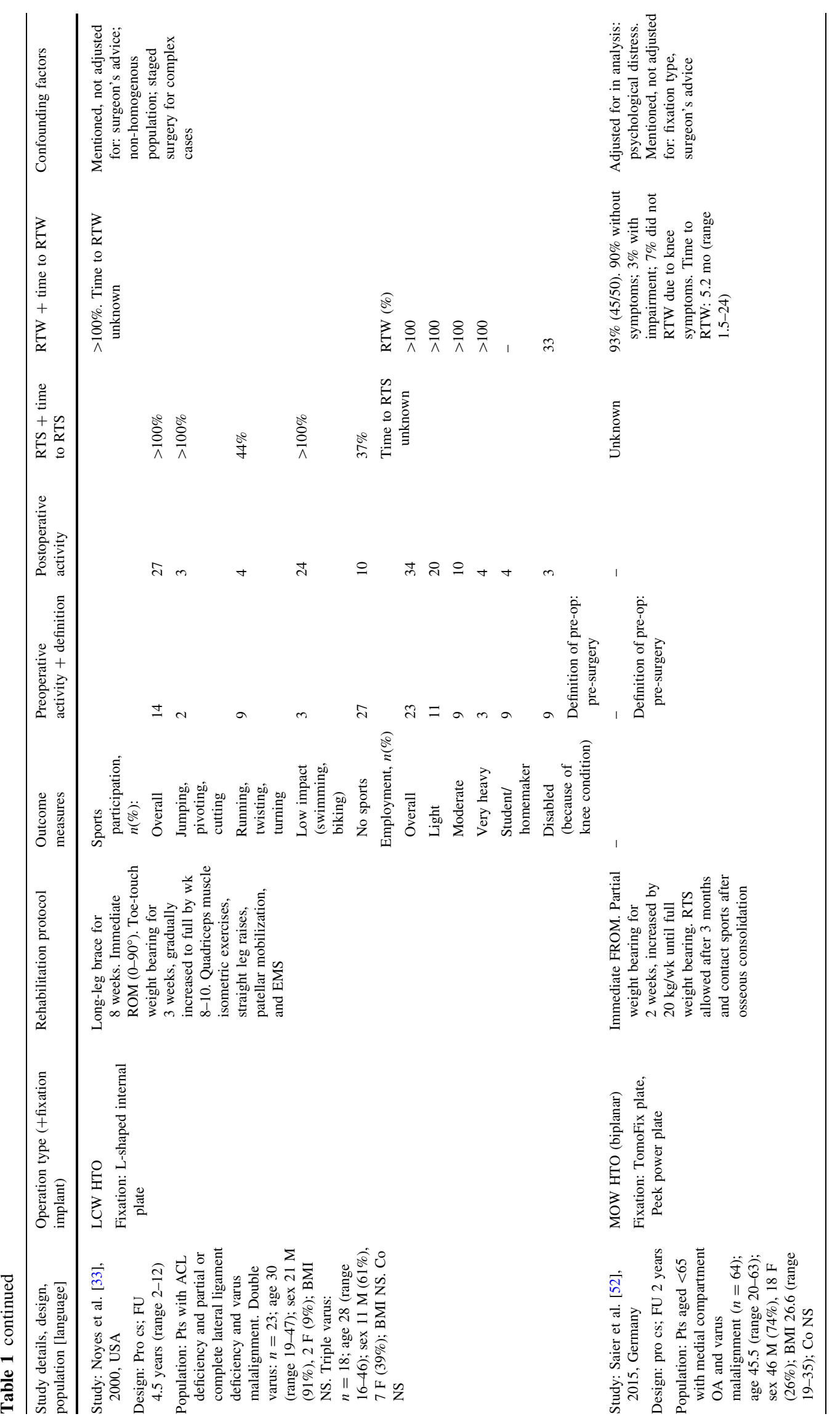




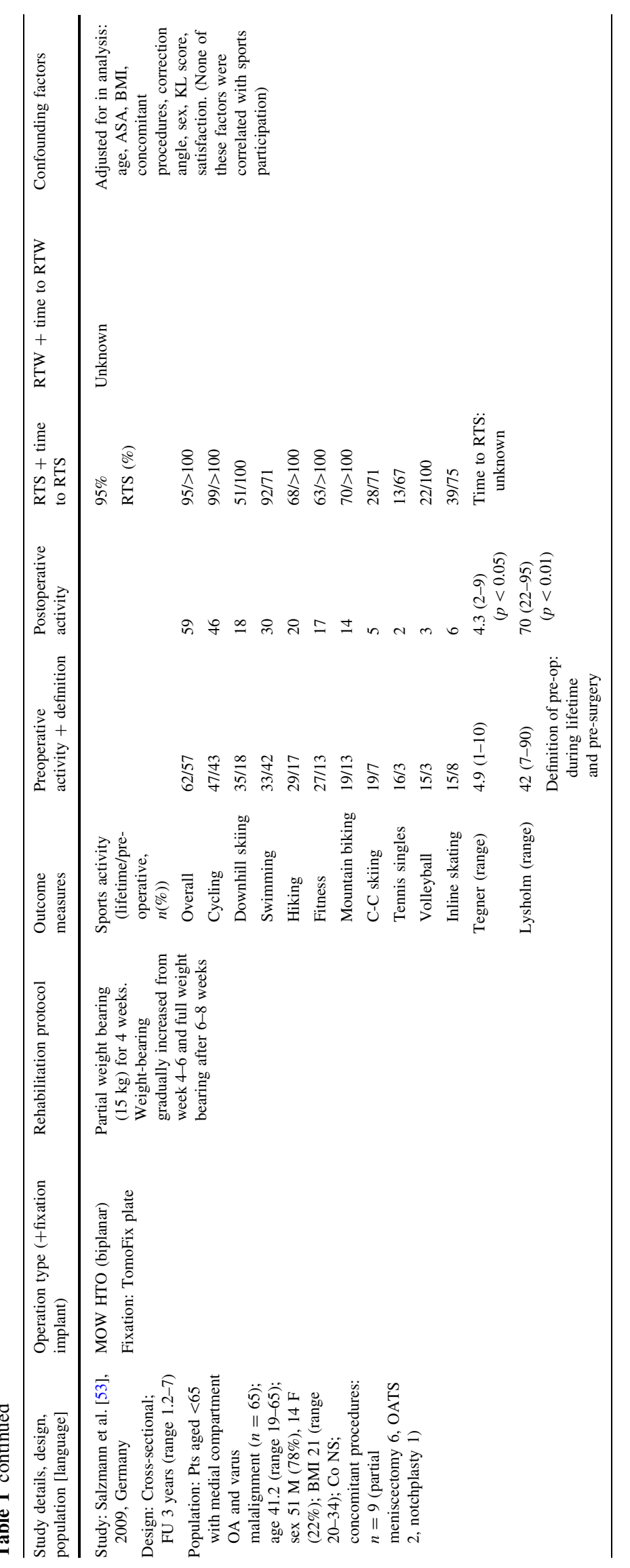




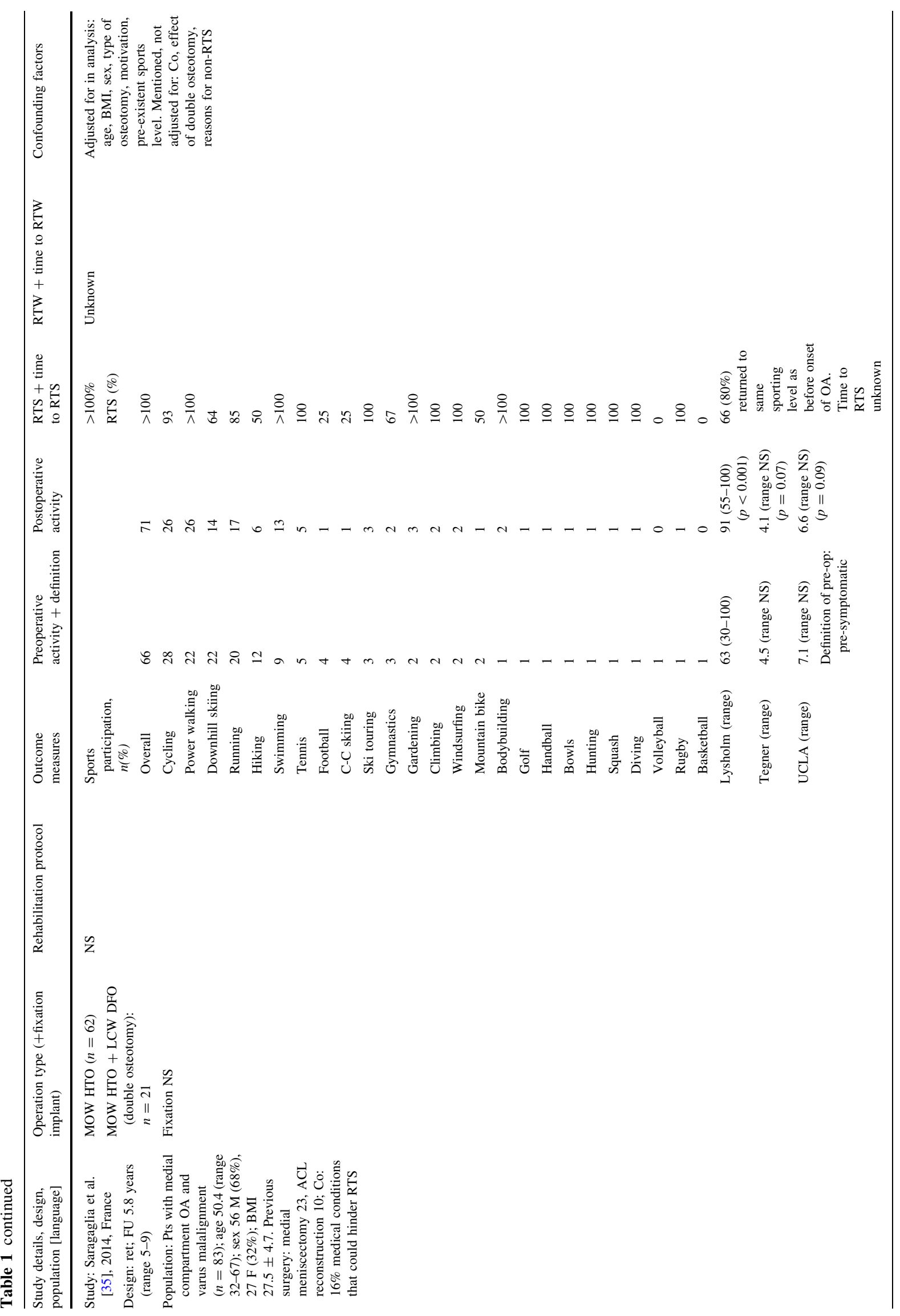




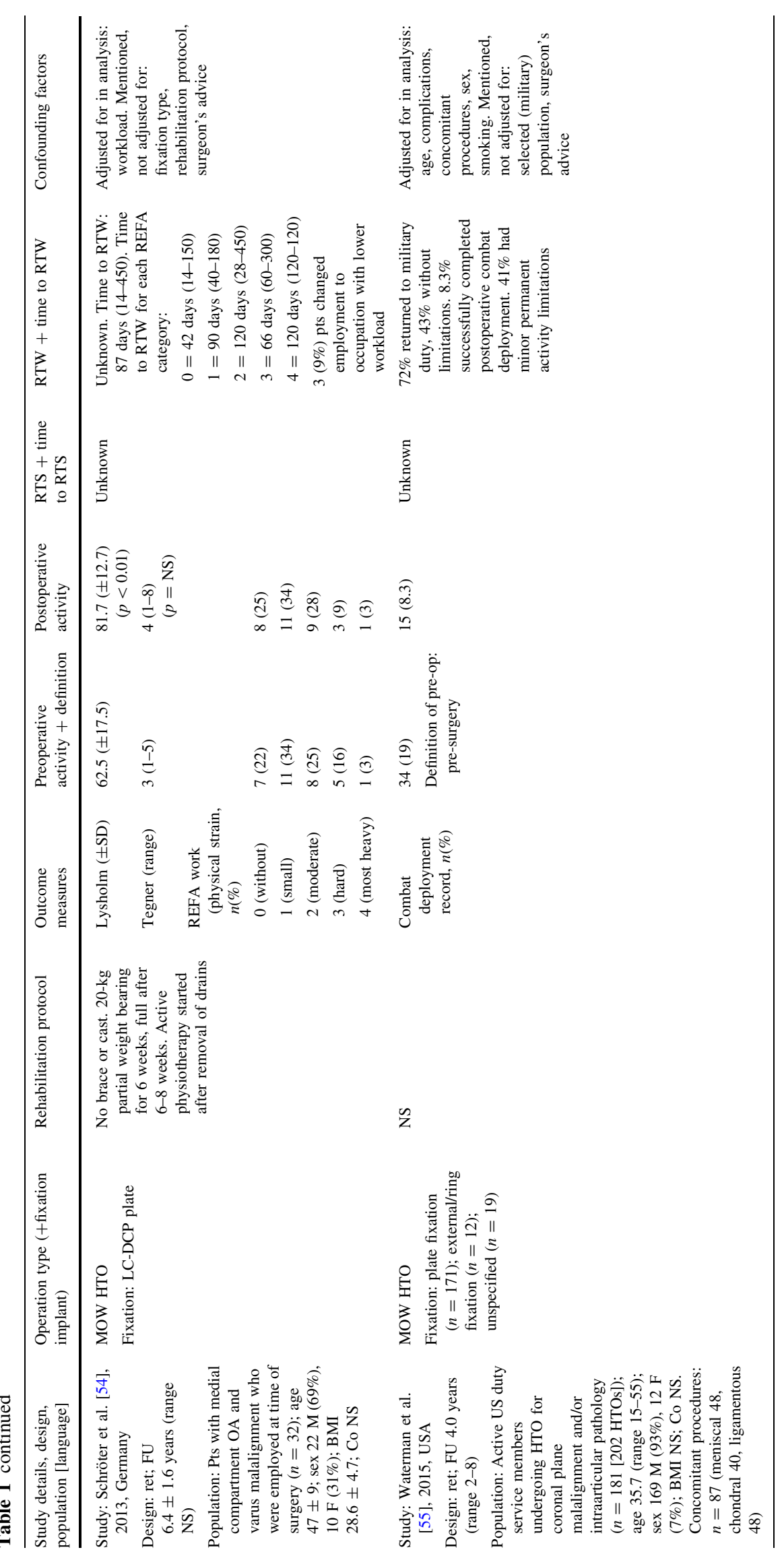




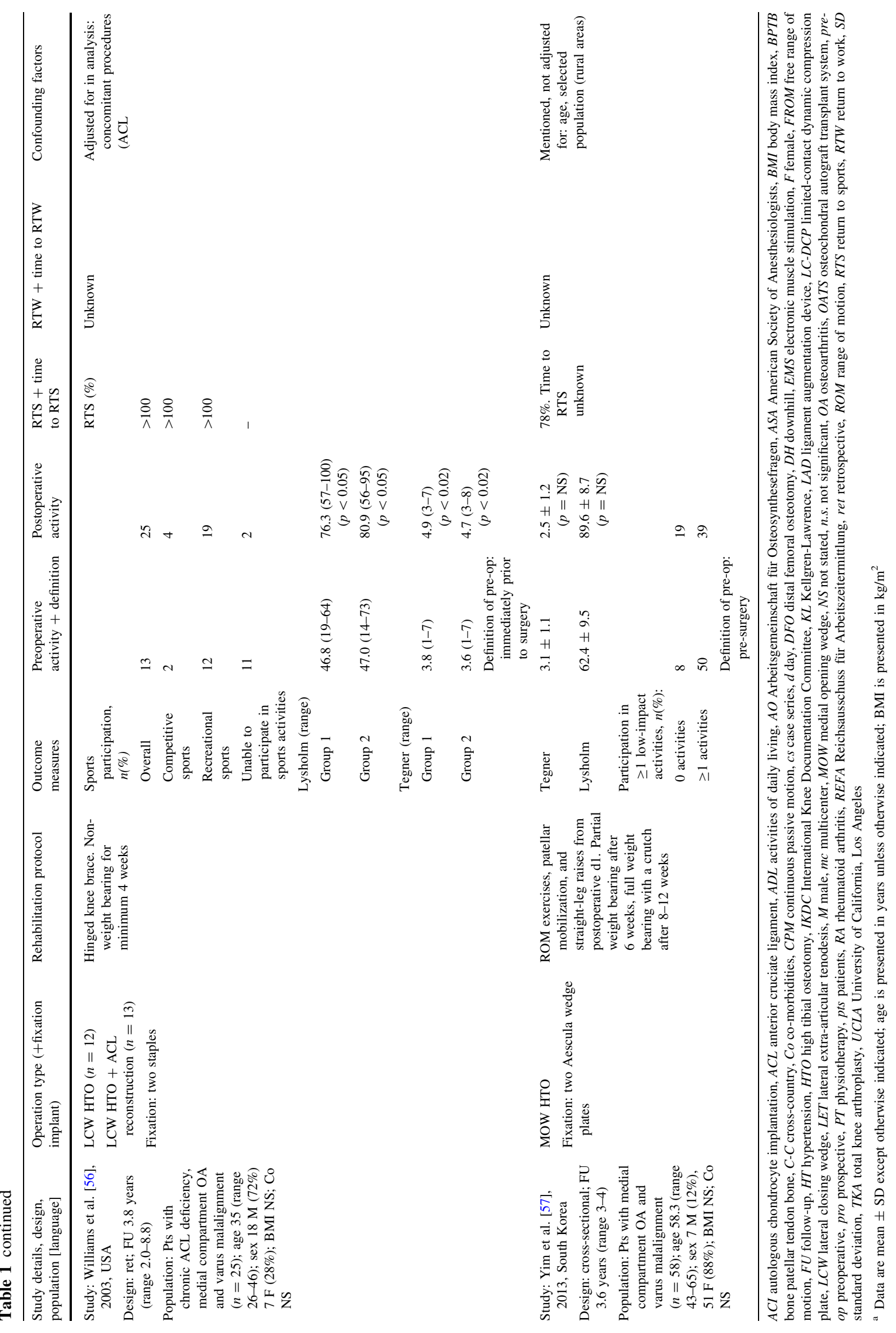




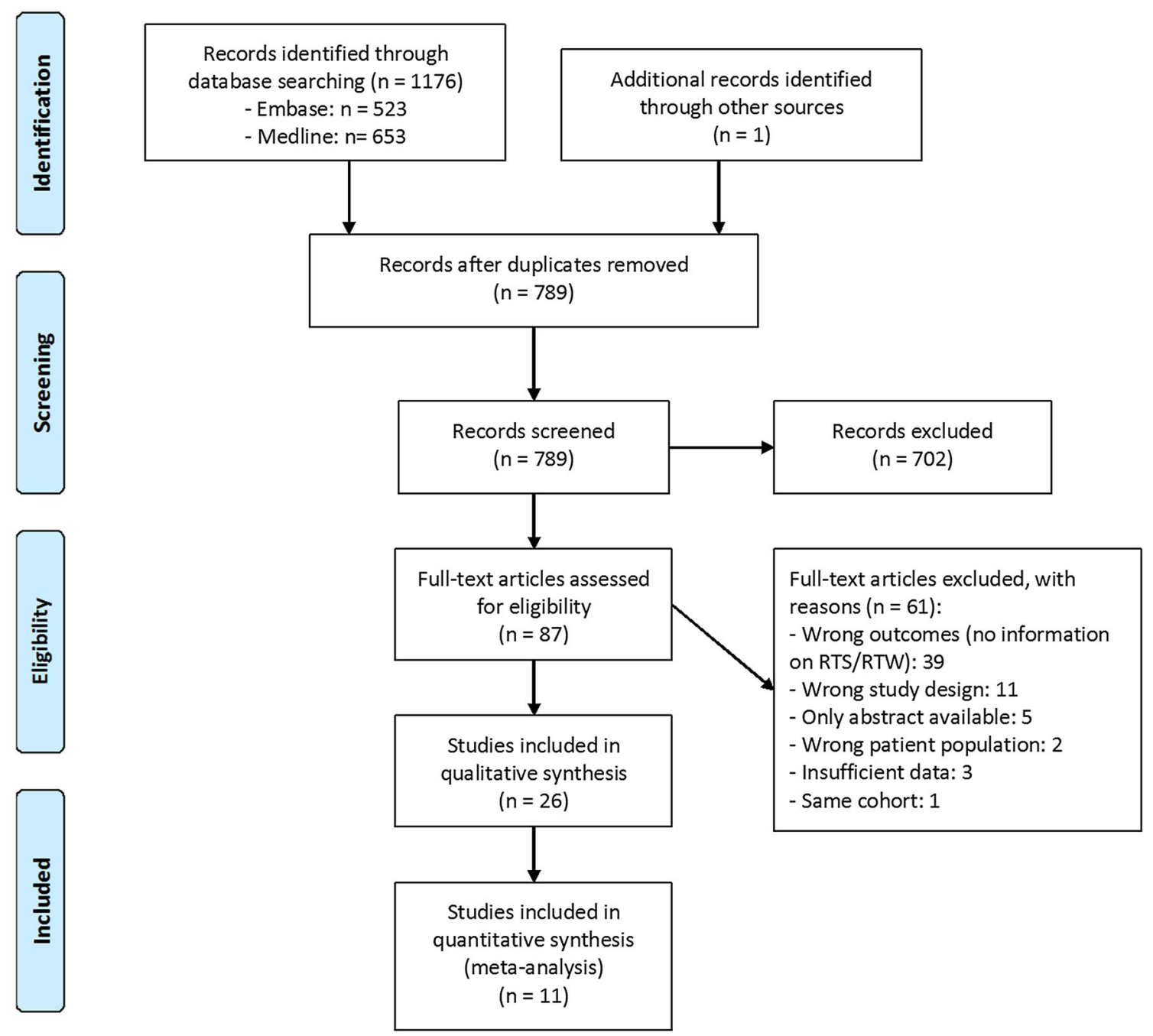

Fig. 1 PRISMA flow diagram

\subsection{Pooling Data}

Data were pooled from the studies that described preand/or postoperative participation in specific types of sports and categorised into low-, intermediate-, or highimpact sports according to the levels of impact on the knee joint (ESM Appendix S3). This classification complies with Vail et al. [31] and is supported by a biomechanical study from Kuster et al. [32], which considered both peak loads and flexion angles of the knee. We calculated pooled RTS percentages by comparing pooled pre- and postoperative sports participation data. In addition, we compared percentages for RTS to the preoperative level and the pre-symptomatic level. We also pooled RTW data for studies that provided preand postoperative work data.

\section{Results}

\subsection{Literature Search}

Figure 1 presents the PRISMA flowchart for our search strategy. Our primary search retrieved 1176 potentially relevant citations. After deleting 387 duplicates, we applied our inclusion criteria to the titles and abstracts of 789 articles. Of the 789 screened articles, disagreement occurred in 45 cases $(6 \%)$, which were all resolved by discussion. This selection yielded 87 potentially relevant full-text articles, which were then reviewed. For the full-text screening, disagreement occurred in four $(5 \%)$ cases, which were resolved by discussion. We subsequently excluded 61 articles for various reasons (Fig. 1). Noyes et al. [33] published two studies involving the same cohort, so we 
only included the study with the longest follow-up. We performed reference screening and forward citation tracking on the remaining articles, which yielded one additional article [34]. Finally, 26 articles were included.

\subsection{Study Characteristics}

\subsubsection{Demographic Data}

Table 1 presents the results of the data extraction. Studies were published between 1983 and 2016, and all the included studies were observational, with four cross-sectional studies, five prospective cohort studies, 14 retrospective cohort studies, and three retrospective case series. One study was performed in Brazil, one in Finland, five in France, nine in Germany, one in Greece, one in Italy, one in South Korea, one in Sweden, one in Switzerland, and five in the USA. The majority of studies were written in English $(n=24)$, one was in French, and one was in Italian. The total number of included patients was 1321 (range 6-181), sex was specified in 24 studies (1251 patients; 857 [69\%] male). Mean age ranged from 27 to 62 years (range 14-80). The mean duration of follow-up was 4.8 years (range 1.8-11.0). Patients' BMI was specified in 12 studies, with mean BMI varying from 21 to $30 \mathrm{~kg} / \mathrm{m}^{2}$. Three of 26 studies included information on comorbidities.

\subsubsection{Surgical Technique}

Nine studies included only medial open-wedge (MOW) HTO, four only lateral closing-wedge (LCW) HTO, six both MOW HTO and LCW HTO, one MOW HTO and MOW HTO + LCW DFO [35], one both MOW HTO and lateral opening-wedge (LOW) DFO [36], one both LCW and medial closing-wedge (MCW) [37], and one LOW DFO [38]. One study reported the use of LCW HTO and a 'Mittelmeier' HTO, which was not further specified [39], one study performed MOW HTO with external fixation (hemicallotasis technique) [40], and one study only mentioned the use of both varising and valgising HTO, but the type was not further specified [41]. For fixation, 20 studies used plate fixation, with six studies using the TomoFix plate, two studies using the Peak-carbon plate, one study using the Puddu plate, and 11 studies using other types of plates (for more details, see Table 1). Seven studies used staples, two studies used external fixators, two studies used plaster casts, and three studies did not describe their fixation method. Concomitant surgery was performed in eight studies, with anterior cruciate ligament (ACL) reconstruction performed in five studies, autologous chondrocyte implantation performed in two studies, and meniscal allograft transplantations performed in one study (Table 1).

\subsection{Methodological Quality}

Overall, 7 of 26 studies scored a low risk of bias, ten studies scored a moderate risk of bias, and nine studies scored a high risk of bias. The lowest risk of bias was found for the prognostic factor domain, describing the type of osteotomy performed and any additional surgery, for which no study scored a high risk of bias. The highest risk of bias was found for the confounding factors (e.g., patient-related factors, surgeons' advice, rehabilitation), with 17 studies scoring a high risk and only four studies scoring a low risk of bias. Table 2 summarizes the methodological assessment for the risk of bias.

\subsection{Return to Sport}

In total, 19 of 26 studies reported the percentage of patients returning to different types of sport activities. Mean RTS percentages varied from 48 to $>100 \%$, with $>100 \%$ indicating that more patients participated in sports activities postoperatively than preoperatively. A definition of preoperative sports participation was provided in 16 of 26 studies. Seven studies describing the preoperative sports level as the moment prior to surgery (pre-surgery level) found RTS varied from 66 to $>100 \%$. Nine studies describing the preoperative sports level as the moment before the onset of knee symptoms (pre-symptomatic level) found that $68-100 \%$ could return to this level. Of the studies with low risk of bias, five provided RTS percentages: $63 \%$ (at 10 years), 78, 92, 100 and $>100 \%$ (more patients participated in sports postoperatively than preoperatively). None of the included studies reported on the timing of RTS.

Data could be pooled for 16 studies that reported exact numbers of patients participating in sports pre- and/or postoperatively. Overall, RTS was $94 \%$, but this depended on how the preoperative sports level was defined (Table 3). Seven studies used the pre-surgery level and found an average RTS of $>100 \%$. Nine studies used the pre-symptomatic level and found an average RTS of $85 \%$. For the studies scoring a low risk of bias, three studies used the pre-surgery level and found an average RTS of $89 \%$. Two studies used the pre-symptomatic level and found an average RTS of $78 \%$. In total, 11 studies reported specific numbers of sports that were practiced pre- and postoperatively (Table 4). Preoperatively, 453 patients practiced an average of 1.9 sports, including $47 \%$ low-impact sports, $35 \%$ intermediate-impact sports and 18\% high-impact sports. Postoperatively, 592 patients practiced an average of 1.9 sports, including 58\% low-impact sports, 32\% intermediate-impact sports and $10 \%$ high-impact sports. Five of 11 pooled studies were rated as having a low risk of bias. In these studies, 204 patients practiced an average of 
Table 2 Methodological assessment according to six domains of potential bias (QUIPS)

\begin{tabular}{|c|c|c|c|c|c|c|c|}
\hline Study $(n=26)$ & $\begin{array}{l}\text { Study } \\
\text { participation }\end{array}$ & $\begin{array}{l}\text { Study } \\
\text { attrition }\end{array}$ & $\begin{array}{l}\text { Prognostic } \\
\text { factor }\end{array}$ & Outcome & $\begin{array}{l}\text { Confounding } \\
\text { factors }\end{array}$ & Analysis & $\begin{array}{l}\text { Overall risk of } \\
\text { bias }^{\mathrm{a}}\end{array}$ \\
\hline Ampollini et al. [42] & Moderate & Low & Low & Moderate & High & Moderate & Moderate \\
\hline Bode et al. [25] & Low & Low & Low & Low & High & Low & Moderate \\
\hline Bonnin et al. [43] & Moderate & High & Moderate & Low & High & Low & High \\
\hline Boss et al. [44] & Moderate & Low & Low & High & High & Low & High \\
\hline Boussaton et al. [41] & Moderate & Low & Moderate & High & High & Moderate & High \\
\hline Cotic et al. [45] & Low & Low & Low & Low & Moderate & Low & Low \\
\hline Dahl et al. [40] & Low & Low & Low & Low & Moderate & Low & Low \\
\hline De Carvalho et al. [38] & Low & Moderate & Low & Moderate & High & Low & Moderate \\
\hline Dejour et al. [46] & Moderate & High & Low & Low & High & High & High \\
\hline $\begin{array}{l}\text { Faschingbauer et al. } \\
\text { [47] }\end{array}$ & Low & Moderate & Low & Low & Moderate & Low & Low \\
\hline Gomoll et al. [36] & Low & Low & Low & Low & High & High & High \\
\hline Hoell et al. [34] & Moderate & Moderate & Low & Low & High & High & High \\
\hline Isolauri et al. [37] & High & High & Moderate & High & High & High & High \\
\hline Korovessis et al. [39] & Low & Moderate & Moderate & Moderate & High & Low & Moderate \\
\hline Lerat et al. [48] & High & High & Moderate & Moderate & High & Low & High \\
\hline Minzlaff et al. [49] & Low & Low & Low & Moderate & Low & Low & Low \\
\hline Nagel et al. [50] & High & High & Low & Low & Moderate & Moderate & High \\
\hline Niemeyer et al. [51] & Low & Low & Low & Low & High & Low & Moderate \\
\hline Noyes et al. [33] & Moderate & Low & Low & Moderate & High & Low & Moderate \\
\hline Saier et al. [52] & Low & Moderate & Low & Low & Low & Low & Low \\
\hline Salzmann et al. [53] & Moderate & Moderate & Low & Moderate & High & Moderate & Moderate \\
\hline Saragaglia et al. [35] & High & Moderate & Low & Moderate & Low & Low & Moderate \\
\hline Schröter et al. [54] & Low & Moderate & Low & Low & High & Low & Moderate \\
\hline Waterman et al. [55] & Low & Low & Moderate & Low & Low & Moderate & Low \\
\hline Williams et al. [56] & Moderate & Moderate & Low & Moderate & High & Moderate & Moderate \\
\hline Yim et al. [57] & Low & Low & Low & Moderate & Moderate & Low & Low \\
\hline
\end{tabular}

QUIPS Quality in Prognosis Studies

${ }^{a}$ We considered a study to be of low risk of bias when the methodological risk of bias was rated as low or moderate on all of the six domains, with at least four rated as low. A study was scored as high risk of bias if two or more of the domains were scored as high

1.9 sports preoperatively, including $55 \%$ low-impact sports, 32\% intermediate-impact sports and 12\% high-impact sports. Postoperatively, 204 patients practiced an average of 1.9 sports, including $56 \%$ low-impact sports, $35 \%$ intermediate-impact sports and 9\% high-impact sports.

\subsection{Return to Work}

In total, 11 of 26 studies reported on the possibility of RTW after HTO (Table 1). Mean RTW varied from 41 to $>100 \%$, with $>100 \%$ indicating that more patients were working postoperatively than preoperatively. For the studies with a low risk of bias, RTW rates were 72, 84, 93 and $94 \%$. One study investigated a military population with a very high workload and found that $72 \%$ could RTW [55]. Another study investigated an agricultural population with a high workload and found that $86 \%$ could RTW [39]. Four studies reported on the timing of RTW, which varied from 9.7 to 22.1 weeks. One additional study reported that $89 \%$ of an homogeneous group of agricultural workers had returned to work after $8-12$ months, but did not specify the exact timing [39]. Two studies found timing of RTW was significantly dependent on the workload, which was assessed using the REFA workload classification [25, 54]. Duration of inability to work varied from 6 and 10 weeks for REFA grade 0 (lowest workload) to 17 and 22 weeks for REFA 4 (heaviest physical strain) $(p<0.05)$. In line with these findings, Faschingbauer et al. [47] found that workers with the highest workload returned after 19.1 weeks and those with the lowest workload returned after 11.8 weeks, although this difference was not statistically significant. In terms of working capacity at follow-up, $72-100 \%$ of patients returned to the same or a higher 
Table 3 Pooled data for numbers of patients participating in any sport pre- and postoperatively

\begin{tabular}{lllr}
\hline Preoperative reference for RTS & $\begin{array}{l}\text { No. of pts participating in any sport } \\
\text { preoperatively }\end{array}$ & \multicolumn{1}{l}{$\begin{array}{l}\text { No. of pts participating in any sport } \\
\text { postoperatively }\end{array}$} & $\begin{array}{l}\text { RTS } \\
(\%)\end{array}$ \\
\hline Overall (16 studies) & 463 & 434 \\
$\begin{array}{l}\text { Pre-surgery status as reference for RTS (7 } \\
\text { studies) }\end{array}$ & 150 & 167 \\
$\begin{array}{l}\text { Pre-symptomatic status as reference for RTS } \\
\quad(9 \text { studies) }\end{array}$ & 313 & 267 \\
Low risk of bias studies (5 studies) & 181 & 141 \\
\hline
\end{tabular}

pts patients, RTS return to sport

Table 4 Pooled data for pre- and postoperative sports participation for different types of sports impact

\begin{tabular}{|c|c|c|c|c|c|c|}
\hline \multirow[t]{2}{*}{ Impact } & \multicolumn{3}{|c|}{$\begin{array}{l}\text { Sports participation preoperatively ( } n=10 \\
\text { studies) }\end{array}$} & \multicolumn{3}{|c|}{$\begin{array}{l}\text { Sports participation postoperatively ( } n=11 \\
\text { studies) }\end{array}$} \\
\hline & $\begin{array}{l}\text { Sports } \\
(n)\end{array}$ & $\begin{array}{l}\text { Patients } \\
(n)\end{array}$ & $\begin{array}{l}\text { Average sports/patient, } \\
n(\%)\end{array}$ & $\begin{array}{l}\text { Sports } \\
(n)\end{array}$ & $\begin{array}{l}\text { Patients } \\
(n)\end{array}$ & $\begin{array}{l}\text { Average sports/patient, } \\
n(\%)\end{array}$ \\
\hline $\begin{array}{l}\text { Low (e.g. cycling, swimming, } \\
\text { golfing) }\end{array}$ & 413 & 453 & $0.91(47)$ & 658 & 592 & $1.11(58)$ \\
\hline $\begin{array}{l}\text { Intermediate (e.g. hiking, downhill } \\
\text { skiing) }\end{array}$ & 303 & 453 & $0.67(35)$ & 369 & 592 & $0.62(32)$ \\
\hline High (e.g. tennis, running, ball sports) & 159 & 453 & $0.35(18)$ & 109 & 592 & $0.18(10)$ \\
\hline Total & 875 & 453 & 1.93 & 1136 & 592 & 1.92 \\
\hline
\end{tabular}

Table 5 Pooled data for return to work and average duration of inability to work

\begin{tabular}{|c|c|c|c|c|c|c|}
\hline \multirow[t]{2}{*}{ Study $(n=7)$} & \multicolumn{3}{|c|}{ Number of working patients } & \multicolumn{3}{|l|}{ Time to RTW } \\
\hline & $\begin{array}{l}\text { Preoperative } \\
(n)\end{array}$ & $\begin{array}{l}\text { Postoperative } \\
\text { (n) }\end{array}$ & RTW (\%) & Study $(n=6)$ & Patients $(n)$ & Inability to work (weeks) \\
\hline Dahl et al. [40] & 43 & 38 & 88 & Bode et al. [25] & 40 & 13.5 \\
\hline De Carvalho et al. [38] & 26 & 23 & 88 & Faschingbauer et al. [47] & 40 & 16.7 \\
\hline Faschingbauer et al. [47] & 43 & 40 & 93 & Hoell $^{\mathrm{a}}$ (ow) et al. [34] & 40 & 13.9 \\
\hline Korovessis et al. [39] & 63 & 54 & 86 & Hoell $^{\mathrm{a}}$ (cw) et al. [34] & 51 & 13.6 \\
\hline Noyes et al. [33] & 23 & 34 & 148 & Lerat et al. [48] & 49 & 20 \\
\hline Saier et al. [52] & 50 & 45 & 90 & Saier et al. [52] & 64 & 20.8 \\
\hline Waterman et al. [55] & 181 & 130 & 72 & Schröter et al. [54] & 32 & 12.4 \\
\hline Total & 429 & 364 & 85 & Total & 276 & 16.3 \\
\hline
\end{tabular}

$R T W$ return to work, $O W$ opening-wedge, $C W$ closing-wedge, $H T O$ high tibial osteotomy

${ }^{a}$ Hoell et al. reported separate duration of inability to work after opening-wedge HTO and closing-wedge HTO

workload. Finally, one study investigating RTW after DFO found that $89 \%$ of patients could RTW [38]. The duration of inability to work was not mentioned.

Data could be pooled for seven studies, including two with a low risk of bias, which reported exact numbers of patients working pre- and postoperatively. Overall, $85 \%$ of patients could RTW (Table 5). In studies with a low risk of bias, $80 \%$ could RTW. Six studies described the duration of inability to work. On average, patients were unable to work for 16 weeks (Table 5). Two studies with a low risk of bias reported that patients were unable to work for an average of 19 weeks. This included the study by Saier et al. [52], who found that, overall, patients were unable to work for 21 weeks. Separate analysis showed that patients with a concomitant mental disorder could RTW after an average of 36 weeks compared with 16 weeks in the mentally healthy group. 


\subsection{Secondary Outcome Measures of Physical Activity}

The Tegner score, Lysholm score, UCLA score, and IKDC score were described as secondary outcome measures for physical activity. IKDC scores $(0-100)$ were used in three studies. Gomoll et al. [36] and Niemeyer et al. [51] described median preoperative scores of 26 and 40 and median postoperative scores of 63 and 70, respectively. Boussaton and Potel [41] described a median postoperative IKDC score of 94 (range 86-99). The Lysholm score was described in 12 studies, with median preoperative scores ranging from 5 to 63 and median postoperative scores ranging from 63 to 91 . The Tegner score was described in 11 studies, with median preoperative scores ranging from 3.1 to 6.5 and median postoperative scores ranging from 2.5 to 5.9. The UCLA score was described in one study, with a median preoperative score of 7.1 and postoperative score of 6.6 [35].

\subsection{Confounders}

We scored whether studies mentioned possible confounders, and whether analyses were adjusted for these confounders. Possible confounders that could influence RTS and/or RTW were mentioned in 25 of 26 studies, but only 15 studies adjusted for one or more confounders in the analysis. Age was mentioned as a possible confounder in 11 studies, and three studies adjusted for it. Minzlaff et al. [49] found that younger patients reached a higher frequency of post-operative sports. In contrast, Salzmann et al. [53] and Saragaglia et al. [35] found age had no influence on RTS. BMI was mentioned as a possible confounder in four studies. Two studies adjusted for BMI but found no influence on RTS. Four studies mentioned sex as a confounder, and three studies adjusted for it but found no effect on RTS. Three studies mentioned comorbidities as a possible confounder. Salzmann et al. [53] adjusted for comorbidities using the American Society of Anesthesiologists classification but found no correlation with RTS. Saragaglia et al. [35] specifically mentioned reasons for patients who could not RTS. Of 12 patients, four had medical contraindications, three had severe intractability, and five indicated that the knee was solely responsible for the inability to RTS. Four studies mentioned concomitant procedures as a possible confounder. Salzmann et al. [53] found no effect of concomitant procedures on RTS, whereas Waterman et al. [55] found that concomitant procedures increased the risk of failure. The influence of patient motivation was mentioned in four studies. Bonnin et al. [43] found motivation to be strongly correlated to RTS, whereas Saragaglia et al. [35] found no correlation. The preoperative sports level was mentioned as a confounder in six studies. Nagel et al. [50] found preoperative sports level to be the most predictive factor for RTS, whereas Saragaglia et al. [35] found no correlation. The influence of the surgeons' advice on RTS was mentioned in nine studies. Most surgeons in these studies advised their patients that RTS was not the goal of surgery and tried to moderate their patients' sporting ambitions. Faschingbauer et al. [47] and Noyes et al. [33] discouraged participation in high-impact activities such as soccer and tennis. The rehabilitation protocol was mentioned in 19 of 26 studies, but the description was often very brief, only including information about the first phase of rehabilitation, concerning range of motion (ROM) and weight-bearing advice. Five studies described their RTS advice in detail. Three studies [36, 44, 52] advised a return to activities of daily life and low-impact sports after 3 months and a return to more demanding activities and contact sports after 6-12 months. Two studies [38, 49] allowed full RTS, including contact sports, after radiologically confirmed healing of the osteotomy.

Finally, three studies adjusted for the effect of workload on RTW: two of these $[25,54]$ found that higher workloads resulted in longer inability to work, but one study [47] found no significant difference in RTW between high and low workloads. Only one study [34] compared RTW for different types of HTO; it found no significant difference in time to RTW between open- and closed-wedge HTO.

\section{Discussion}

Our systematic review showed that a large percentage of patients were able to RTS activities and RTW after osteotomies around the knee. Concerning sports activities, 66 to $>100 \%$, with $>100 \%$ indicating more patients participated in sports postoperatively than preoperatively, of patients could RTS. An overall trend was observed towards participation in lower-impact activities after surgery. The diversity in RTS percentages was mostly caused by the different definitions used for the preoperative reference point for sports participation. Remarkably, none of the included studies reported on the timing of RTS. Concerning RTW, 41 to $>100 \%$ of patients could RTW and $72-100 \%$ of patients could return to the same or a higher workload. The duration of inability to work varied from 10 to 22 weeks.

\subsection{Return to Sport}

The meta-analysis showed that overall, $94 \%$ of patients could RTS, and $85 \%$ returned to their pre-symptomatic sports level after knee osteotomies. In a recent review on RTS and RTW after HTO, Ekhtiari et al. [58] found that $87 \%$ could RTS. However, the authors did not take into 
account the definition of preoperative sports participation, and our review showed that different definitions resulted in considerable variance in RTS percentages. Moreover, Ekhtiari et al. [58] only evaluated results of RTS and RTW after HTO, described in ten studies, including 250 patients, whereas we reviewed results after any osteotomy around the knee and found 16 studies, including 463 patients. Lastly, the indication for HTO was knee OA in almost all studies in their review. We observed that osteotomies around the knee are also increasingly performed for other indications, such as in addition to ligament reconstruction or articular cartilage restoration procedures. Such patients are often younger and thus more likely to wish to return to more demanding activities. For these patients in particular, it is imperative to know whether it is possible to RTS and RTW.

In a review of RTS after KA, Witjes et al. [6] found that 36-89\% could RTS after total KA (TKA), and 74 to $>100 \%$ could RTS following unicondylar KA (UKA). Postoperatively, patients undergoing TKA were engaged in an average of 1.0 sports, including $87 \%$ low-impact sports, 9\% intermediate-impact sports, and 4\% high-impact sports. Patients undergoing UKA were engaged in an average of 1.5 sports, including 77\% low-impact sports, $19 \%$ intermediate-impact sports, and $4 \%$ high-impact sports. The present study demonstrates that patients participated in an average of 1.9 sports postoperatively, including 58\% lowimpact sports, $32 \%$ intermediate-impact sports, and $10 \%$ high-impact sports. Thus, on average, patients undergoing knee osteotomies returned to more sports than did patients undergoing KA. A shift to participation in lower-impact sports activities was observed in all three groups, but highimpact sports were performed more often after knee osteotomy than after KA. Thus, the possibility of returning to high-impact sports appears most likely after knee osteotomies and is also possible, though less likely, after UKA. In contrast, participation in high-impact sports after TKA is most unlikely. However, these findings could, at least in part, be explained by the generally younger age and less severe grades of knee OA in patients undergoing knee osteotomy compared with those undergoing KA.

\subsubsection{Factors Influencing Return to Sport}

The existing evidence on factors that influence RTS after knee osteotomy is ambiguous. Nagel et al. [50] found that the most predictive factor for RTS after HTO was the patient's preoperative sporting level. Patient motivation appears to be another important factor. Mancuso et al. [59] found that only $30 \%$ of patients undergoing TKA expressed motivation to RTS, whereas Saragaglia et al. [35] found that $71 \%$ of patients undergoing HTO were motivated to RTS but that neither the motivation nor the pre-existent sport level was related to greater RTS. In contrast, Bonnin et al. [43] found a correlation between patient motivation and activity level, with motivated patients being more active postoperatively. These contrasting findings may be explained by the nature of the practiced sports. Despite high motivation, a return to high-impact sports is more difficult than a return to low-impact sports. Comorbidities that could possibly hinder patients in their RTS were only described in 3 of 26 studies. One study [35] found that 12 of 83 patients could not RTS because of comorbidities, and knee symptoms were solely responsible for the inability to RTS in five patients. Thus, we cannot rule out that specific medical conditions unrelated to the knee surgery had a negative influence on the number of patients that could RTS and RTW in other studies.

Our results confirm that, when assessing RTS, it is very important to use a clear definition of the preoperative sports level (e.g., preoperative, pre-symptomatic), as previously stated by Witjes et al. [6]. Remarkably, only 18 studies reported their definition, and only nine studies used the presymptomatic sports level to calculate RTS percentages. A return to pre-surgery sports level was possible in $>100 \%$, whereas a return to the pre-symptomatic level was possible in only $85 \%$. We believe that the pre-symptomatic level is most relevant for young, active patients, since it is conceivable that this patient population in particular expects to return to the activities they performed before the onset of knee symptoms.

Finally, evidence on the return to professional or competitive levels of sports after knee osteotomies is sparse. A French study by Boussaton and Potel [41] followed six professional rugby players who all successfully returned to play, with follow-up varying from 1 to 10 years. Faschingbauer et al. [47] included four competitive-level athletes: two football players, one rugby player, and one squash player. Only one athlete, the rugby player, could return to competitive sport. In the study by Williams et al. [56], two patients participated in (unspecified) competitive sports preoperatively, whereas four patients were participating in competitive sports at a mean follow-up of 3.8 years. Lerat et al. [48] found that two of ten patients could return to competitive boxing and tennis, respectively. We found one other review describing two cases of National Football League players who successfully returned to play after HTO [26]. Still, the authors highlighted that, even in elite athletes, the goal of HTO is not resumption of competition but rather to allow daily and recreational-level activities. This consideration is in line with the surgeons' advice that was described in nine of the studies included in this review. However, even without taking into account the effect of possibly discouraging advice from surgeons, our results show that a reasonable number of patients are able to successfully return to high- 
impact sports activities. Therefore, we believe that a return to competitive sports should not be ruled out in advance. As indicated, native knee structures are spared in knee osteotomies, without any risk of wear to a prosthesis. Thus, when full consolidation of the osteotomy is achieved, a return to competitive sports may be attempted. However, this also depends on the original indication for the osteotomy. Expectations of RTS may need to be tempered based on the indication.

\subsection{Return to Work}

This review is the first to systematically assess the possibility of RTW after all types of knee osteotomies. We found that 364 of $429(85 \%)$ patients could RTW and that the mean duration of their inability to work was 16.3 weeks. This is in line with the aforementioned review by Ekhtiari et al. [58], who found 310 of 367 (85\%) patients could RTW. Based on existing studies, we cannot draw definite conclusions on the possibility of returning to the same or higher workloads. However, our findings do indicate that a RTW with high workloads (e.g., military service, work with heavy physical strain) is less likely than a RTW with low workloads.

\subsubsection{Factors Influencing Return to Work}

Our study is the first to describe factors influencing RTW after knee osteotomies. Such factors have been described before in patients undergoing KA and included a job with high physical demands on the knee, preoperative sick leave, and patient movement restrictions [60-62]. It seems reasonable that patients with physically demanding jobs need more time to RTW. Of the three studies we included that adjusted for workload, two found that higher workloads resulted in significantly longer inability to work $[25,54]$, but one study did not find this association [47]. Unfortunately, data on preoperative sick leave were not available for any of the included studies. Thus, more studies with larger patient groups are needed to clarify the relationship between these factors and RTW after knee osteotomy. Finally, the influence of movement restrictions could be partly compared between studies using the weight-bearing advice, which may influence the possibility of RTW. Immediate weight-bearing can allow for an earlier return to activities, including work. Recently, Lansdaal et al. [63] showed that immediate full weight-bearing compared with delayed full weight-bearing ( 2 months) after HTO with TomoFix plate fixation was safe and did not compromise functional outcome. The use of angle-stable fixation plates, such as the TomoFix plate, offers superior initial stability compared with other plates, and immediate weight-bearing is possible with this type of plate fixation [64]. Of six studies reporting on time to RTW, three used the TomoFix plate, one used the Association for the Study of Internal Fixation (AO) L-plate, one used the Puddu plate and/or staples, and one used an unspecified plate and/or staples. Only Saier et al. [52] and Faschingbauer et al. [47] reported the use of an early weight-bearing protocol after 2 weeks, and both studies used the TomoFix plate for fixation. Interestingly, the average time to RTW in the study by Saier et al. [52] was the longest of all included studies (21 weeks), whereas Faschingbauer et al. [47] reported an average of 17 weeks. The other studies reported 6-8 weeks of partial weight-bearing and found an inability to work of 12-20 weeks. Based on this evidence, we therefore cannot confirm or reject the hypothesis that using plates that allow early weight-bearing results in earlier RTW. Saier et al. [52] attributed their findings of a late RTW to the presence of mental disorder in the included patients, because separate analysis showed that patients with mental disorder took considerably longer to RTW than mentally healthy patients (36 vs. 16 weeks, respectively, on average). This emphasizes the importance of recognizing another important confounder, namely mental disorders, a known risk factor for worse outcome after knee surgery [65].

\subsection{Strengths and Limitations}

One strength of the present systematic review is that we included all osteotomies around the knee and studies of all indications for osteotomies. Waterman et al. [55] observed that concomitant chondral restoration, meniscal and ligamentous procedures were performed in nearly half of 181 HTOs in a young military population. We believe that the use of osteotomies as an adjunct to reconstructive knee procedures in young, highly active patients will continue to increase. Therefore, it is important to be able to counsel these patients on the possibility of resuming high-demand activities, thus, we also included studies concerning these other osteotomy indications.

A limitation common to any systematic review is the risk of overlooking papers. However, we tried to overcome this with our extensive search strategy, which was conducted by an experienced clinical librarian (JD). Furthermore, we imposed no language restrictions and included French and Italian articles. A specific limitation to our systematic review is that the included studies showed a broad heterogeneity in terms of study design, study population, outcome measures, and overall quality. Thus, while this review presents the best available evidence on RTS and RTW after knee osteotomy, our results should be interpreted with caution. For example, preoperative or presymptomatic sports levels and work participation data were mostly collected postoperatively, which makes these findings prone to recall bias. Furthermore, many different secondary outcome measures for physical activity were 
used (e.g., Tegner score, Lysholm score, UCLA score), hampering comparisons of physical activity between studies. In addition, only a few studies corrected for confounding. For example, only 10 of 26 studies reported the mean BMI. This appears to be an important confounder since BMI $>27.5 \mathrm{~kg} / \mathrm{m}^{2}$ has been associated with worse outcomes, including worse activity levels, after knee osteotomies [66]. This implies that confounders that were not accounted for in the included studies may have influenced our findings. Future prospective studies should identify important confounders such as physical and mental comorbidities, preoperative sports levels and work status, patients' motivation, and surgeon's advice, and should correct for these confounders in the analysis. Also, based on our extensive evaluation of the risk of bias, we found that studies with a low risk of bias reported lower percentages of RTS and RTW. This implies that future studies should carefully consider potential sources of bias and aim to account for these sources in the study design to find the most reliable percentages of RTS and RTW.

\section{Conclusion}

The majority of patients undergoing knee osteotomy return to sports activities and work. For RTS, we observed a trend towards participation in lower-impact sports activities, similar to RTS after KA. Patients undergoing knee osteotomy returned to high-impact activities more often than did those undergoing KA.

For RTW, it appears that a return to the same or a higher workload is possible. This valuable information will aid both the orthopedic surgeon and the patient in the preoperative decision-making process, and is especially interesting in the treatment of the younger, active, and employed OA population. The systematic comparison of current literature is hampered by the heterogeneity of patient populations, operative techniques, and an overall lack of accounting for possible confounding factors. Lastly, this review confirms the importance of using the presymptomatic level as a starting point when analyzing percentages of RTS and RTW.

Acknowledgements The authors would like to thank Dr. F. R. Noyes, Dr. A. Ampollini, Dr. L. A. Pinczewski, Dr. J. P. Roe, Dr. J.L. Lerat, Dr. A. Sternheim, Dr. R. F. Warren, Dr. P. Korovessis, and Dr. T. L. Wickiewicz for kindly providing us with full-text versions of their articles. Our thanks also go to Lucas Benjamin Weterings for his help with translation.

\section{Compliance with Ethical Standards}

Funding No sources of funding were used to assist in the preparation of this article.
Conflict of interest Alan Getgood has received research Grant support from SBM Inc. and consulting fees from Depuy Synthes in regards to osteotomy teaching. Alexander Hoorntje, Suzanne Witjes, Paul Kuijer, Koen Koenraadt, Rutger van Geenen, Joost Daams, and Gino Kerkhoffs have no potential conflicts of interest relevant to the content of this review.

Open Access This article is distributed under the terms of the Creative Commons Attribution 4.0 International License (http:// creativecommons.org/licenses/by/4.0/), which permits unrestricted use, distribution, and reproduction in any medium, provided you give appropriate credit to the original author(s) and the source, provide a link to the Creative Commons license, and indicate if changes were made.

\section{References}

1. Hofmann S, Lobenhoffer P, Staubli A, et al. Osteotomien am kniegelenk bei monokompartmentarthrose. Orthopade. 2009;38:755-70.

2. McNamara I, Birmingham TB, Fowler PJ, et al. High tibial osteotomy: evolution of research and clinical applications: a Canadian experience. Knee Surg Sports Traumatol Arthrosc. 2013;21(1):23-31.

3. Coventry MB. Osteotomy of the upper portion of the tibia for degenerative arthritis of the knee. A preliminary report. J Bone Joint Surg Am. 1965;47:984-90.

4. Brinkman J-M, Lobenhoffer P, Agneskirchner JD, et al. Osteotomies around the knee. J Bone Joint Surg Br. 2008;90B:1548-57.

5. Niinimäki TT, Eskelinen A, Ohtonen $\mathrm{P}$, et al. Incidence of osteotomies around the knee for the treatment of knee osteoarthritis: a 22-year population-based study. Int Orthop. 2012;36:1399-402.

6. Witjes S, Gouttebarge V, Kuijer PPFM, et al. Return to sports and physical activity after total and unicondylar knee arthroplasty: a systematic review and meta-analysis. Sports Med. 2016;46:1-24.

7. Schmalzried TP, Shepherd EF, Dorey FJ, et al. The John Charnley Award. Wear is a function of use, not time. Clin Orthop Relat Res. 2000;381:36-46.

8. Kurtz SM, Lau E, Ong K, et al. Future young patient demand for primary and revision joint replacement: National projections from 2010 to 2030. Clin Orthop Relat Res. 2009;467:2606-12.

9. Losina E, Thornhill TS, Rome BN, et al. The dramatic increase in total knee replacement utilization rates in the United States cannot be fully explained by growth in population size and the obesity epidemic. J Bone Joint Surg Am. 2012;94:201-7.

10. Lobenhoffer P, Agneskirchner JD. Improvements in surgical technique of valgus high tibial osteotomy. Knee Surg Sports Traumatol Arthrosc. 2003;11:132-8.

11. Brouwer RW, Huizinga MR, Duivenvoorden T, et al. Osteotomy for treating knee osteoarthritis. Cochrane Database Syst Rev. 2014;(12):CD004019.

12. Schallberger A, Jacobi $M$, Wahl $P$, et al. High tibial valgus osteotomy in unicompartmental medial osteoarthritis of the knee: a retrospective follow-up study over 13-21 years. Knee Surg Sports Traumatol Arthrosc. 2011;19:122-7.

13. Niinimäki TT, Eskelinen A, Mann BS, et al. Survivorship of high tibial osteotomy in the treatment of osteoarthritis of the knee: Finnish registry-based study of 3195 knees. J Bone Joint Surg Br. 2012;94:1517-21.

14. Bode G, von Heyden J, Pestka J, et al. Prospective 5-year survival rate data following open-wedge valgus high tibial osteotomy. Knee Surg Sports Traumatol Arthrosc. 2015;23:1949-55. 
15. Efe T, Ahmed G, Heyse TJ, et al. Closing-wedge high tibial osteotomy: survival and risk factor analysis at long-term follow up. BMC Musculoskelet Disord. 2011;12:46.

16. Saithna A, Kundra R, Getgood A, et al. Opening wedge distal femoral varus osteotomy for lateral compartment osteoarthritis in the valgus knee. Knee. 2014;21:172-5.

17. Cameron JI, McCauley JC, Kermanshahi AY, et al. Lateral opening-wedge distal femoral osteotomy: pain relief, functional improvement, and survivorship at 5 years. Clin Orthop Relat Res. 2015;473:2009-15.

18. Backstein D, Morag G, Hanna S, et al. Long-term follow-up of distal femoral varus osteotomy of the knee. J Arthroplasty. 2007;22:2-6.

19. Sternheim A, Garbedian S, Backstein D. Distal femoral varus osteotomy: unloading the lateral compartment: long-term followup of 45 medial closing wedge osteotomies. Orthopedics. 2011;34:e488-90.

20. Finkelstein JA, Gross AE, Davis A. Varus osteotomy of the distal part of the femur. A survivorship analysis. J Bone Joint Surg Am. 1996;78:1348-52.

21. Amendola A, Bonasia DE. Results of high tibial osteotomy: review of the literature. Int Orthop. 2010;34:155-60.

22. Cantin O, Magnussen RA, Corbi F, et al. The role of high tibial osteotomy in the treatment of knee laxity: a comprehensive review. Knee Surg Sports Traumatol Arthrosc. 2015;23:3026-37.

23. Dean CS, Liechti DJ, Chahla J, et al. Clinical outcomes of high tibial osteotomy for knee instability: a systematic review. Orthop J Sport Med. 2016;4:1-9.

24. Zaffagnini S, Bonanzinga T, Grassi A, et al. Combined ACL reconstruction and closing-wedge HTO for varus angulated ACLdeficient knees. Knee Surg Sports Traumatol Arthrosc. 2013;21:934-41.

25. Bode G, Ogon P, Pestka J, et al. Clinical outcome and return to work following single-stage combined autologous chondrocyte implantation and high tibial osteotomy. Int Orthop. 2015;39:689-96.

26. Warme B, Aalderink $\mathrm{K}$, Amendola A. Is there a role for high tibial osteotomies in the athlete? Sports Health. 2011;3:59-69.

27. Johnstone SF, Tranovich MJ, Vyas D, et al. Unicompartmental arthritis in the aging athlete: osteotomy and beyond. Curr Rev Musculoskelet Med. 2013;6:264-72.

28. Moher D, Shamseer L, Clarke M, et al. Preferred reporting items for systematic review and meta-analysis protocols (PRISMA-P) 2015 statement. Syst Rev. 2015;4:1.

29. Ouzzani M, Hammady H, Fedorowicz Z, et al. Rayyan: a web and mobile app for systematic reviews. Syst Rev. 2016;5:210.

30. Hayden JA, Van der Windt DA, Cartwright JL, et al. Research and reporting methods annals of internal medicine assessing bias in studies of prognostic factors. Ann Intern Med. 2013;144:427-37.

31. Vail T, Mallon W, Liebelt R. Athletic activities after joint arthroplasty. Sports Med Arthrosc. 1996;4:298-305.

32. Kuster MS, Spalinger E, Blanksby BA, et al. Endurance sports after total knee replacement: a biomechanical investigation. Med Sci Sports Exerc. 2000;32:721-4.

33. Noyes FR, Barber SD, Simon R. High tibial osteotomy and ligament reconstruction in varus angulated, anterior cruciate ligament-deficient knees. A two- to seven-year follow-up study. Am J Sports Med. 2000;21:2-12.

34. Hoell S, Suttmoeller J, Stoll V, et al. The high tibial osteotomy, open versus closed wedge, a comparison of methods in 108 patients. Arch Orthop Trauma Surg. 2005;125:638-43.

35. Saragaglia D, Rouchy RC, Krayan A, et al. Return to sports after valgus osteotomy of the knee joint in patients with medial unicompartmental osteoarthritis. Int Orthop. 2014;38:2109-14.
36. Gomoll AH, Kang RW, Chen AL, et al. Triad of cartilage restoration for unicompartmental arthritis treatment in young patients. J Knee Surg. 2009;22:137-41.

37. Isolauri J, Lapinsuo M, Aho $\mathrm{H}$, et al. Proximal osteotomy of the tibia in the treatment of osteoarthritis of the knee. Arch Orthop Trauma Surg. 1983;102(2):107-10.

38. de Carvalho L, Temponi E, Soares L, et al. Physical activity after distal femur osteotomy for the treatment of lateral compartment knee osteoarthritis. Knee Surg Sports Traumatol Arthrosc. 2014;22:1607-11.

39. Korovessis P, Katsoudas G, Salonikides P, et al. Medium- and long-term results of high tibial osteotomy for varus gonarthrosis in an agricultural population. Orthopedics. 1999;22:729-36.

40. Dahl A, Toksvig-Larsen S, Lindstrand A. Ten-year results of physical activity after high tibial osteotomy in patients with knee osteoarthritis. Knee Surg Sports Traumatol Arthrosc. 2015;25:902-9.

41. Boussaton M, Potel J-F. L'ostéotomie tibiale chez le rugbyman professionnel. À propos de six cas. J Traumatol Sport. 2007;24:200-2.

42. Ampollini A, Affanni M, D'Angelo G, et al. Tibial osteotomy of valgus combined with intraarticular plastic surgery in evolute anterior laxity [Italian]. Minerva Ortop Traumatol. 1998;49:127-34.

43. Bonnin MP, Laurent JR, Zadegan F, et al. Can patients really participate in sport after high tibial osteotomy? Knee Surg Sports Traumatol Arthrosc. 2013;21:64-73.

44. Boss A, Stutz G, Oursin C, et al. Anterior cruciate ligament reconstruction combined with valgus tibial osteotomy (combined procedure). Knee Surg Sports Traumatol Arthrosc. 1995;3:187-91.

45. Cotic M, Vogt S, Feucht MJ, et al. Prospective evaluation of a new plate fixator for valgus-producing medial open-wedge high tibial osteotomy. Knee Surg Sports Traumatol Arthrosc. 2015;23:3707-16.

46. Dejour H, Neyret P, Boileau P, et al. Anterior cruciate reconstruction combined with valgus tibial osteotomy. Clin Orthop Relat Res. 1994;299:220-8.

47. Faschingbauer M, Nelitz M, Urlaub S, et al. Return to work and sporting activities after high tibial osteotomy. Int Orthop. 2015;39:1527-34.

48. Lerat J, Moyen B, Garin C, et al. Anterior laxity and medial arthritis in varus knee. Reconstruction of the anterior cruciate ligament associated with a high tibial osteotomy. J Orthop Surg. 1993;7:333-42.

49. Minzlaff P, Feucht MJ, Saier T, et al. Can young and active patients participate in sports after osteochondral autologous transfer combined with valgus high tibial osteotomy? Knee Surg Sports Traumatol Arthrosc. 2016;24:1594-600.

50. Nagel A, Insall JN, Scuderi GR. Proximal tibial osteotomy: a subjective outcome study. J Bone Joint Surg. 1996;78:1353-8.

51. Niemeyer P, Koestler W, Kaehny C, et al. Two-year results of open-wedge high tibial osteotomy with fixation by medial plate fixator for medial compartment arthritis with varus malalignment of the knee. Arthroscopy. 2008;24:796-804.

52. Saier T, Minzlaff $P$, Feucht MJ, et al. Health-related quality of life after open-wedge high tibial osteotomy. Knee Surg Sports Traumatol Arthrosc. 2015;25:934-42.

53. Salzmann GM, Ahrens P, Naal FD, et al. Sporting activity after high tibial osteotomy for the treatment of medial compartment knee osteoarthritis. Am J Sport Med. 2009;37:312-8.

54. Schröter S, Mueller J, van Heerwaarden R, et al. Return to work and clinical outcome after open wedge HTO. Knee Surg Sports Traumatol Arthrosc. 2013;21:213-9. 
55. Waterman BR, Hoffmann JD, Laughlin MD, et al. Success of high tibial osteotomy in the United States military. Orthop J Sport Med. 2015;3:2325967115574670.

56. Williams R III, Kelly B, Wickiewicz T, et al. The short-term outcome of surgical treatment for painful varus arthritis in association with chronic ACL deficiency. J Knee Surg. 2003;16:9-16.

57. Yim J-H, Song E-K, Seo H-Y, et al. Comparison of high tibial osteotomy and unicompartmental knee arthroplasty at a minimum follow-up of 3 years. J Arthroplasty. 2013;28:243-7.

58. Ekhtiari S, Haldane CE, de Sa D, et al. Return to work and sport following high tibial osteotomy: a systematic review. J Bone Joint Surg Am. 2016;98:1568-77.

59. Mancuso C, Sculco T, Wickiewicz T, et al. Patients' expectations of knee surgery. J Bone Joint Surg Am. 2001;83-A:1005-12.

60. Tilbury C, Leichtenberg CS, Tordoir RL, et al. Return to work after total hip and knee arthroplasty: results from a clinical study. Rheumatol Int. 2015;35:2059-67.

61. Kuijer PPFM, De Beer MJPM, Houdijk JHP, et al. Beneficial and limiting factors affecting return to work after total knee and hip arthroplasty: a systematic review. J Occup Rehabil. 2009; 19:375-81.

62. Kuijer PPFM, Kievit AJ, Pahlplatz TMJ, et al. Which patients do not return to work after total knee arthroplasty? Rheumatol Int. 2016;36:1-6.

63. Lansdaal JR, Mouton T, Wascher DC, et al. Early weight bearing versus delayed weight bearing in medial opening wedge high tibial osteotomy: a randomized controlled trial. Knee Surg Sports Traumatol Arthrosc. (Epub 1 July 2016).

64. Brinkman JM, Luites JW, Wymenga AB, et al. Early full weight bearing is safe in open-wedge high tibial osteotomy. Acta Orthop. 2010;81:193-8.

65. Khatib Y, Madan A, Naylor JM, et al. Do psychological factors predict poor outcome in patients undergoing TKA? A systematic review. Clin Orthop Relat Res. 2015;473:2630-8.

66. Akizuki S, Shibakawa A, Takizawa T, et al. The long-term outcome of high tibial osteotomy: a ten- to 20-year follow-up. J Bone Joint Surg Br. 2008;90:592-6. 